ESAIM: M2AN 47 (2013) 1367-1386

DOI: $10.1051 / \mathrm{m} 2 \mathrm{an} / 2013073$
ESAIM: Mathematical Modelling and Numerical Analysis

www.esaim-m2an.org

\title{
MODÈLE EFFECTIF DE COUCHE MINCE RUGUEUSE PÉRIODIQUE SUR UNE STRUCTURE SEMI-INFINIE
}

\author{
JeAn-Baptiste Bellet ${ }^{1}$ et GÉrard Berginc ${ }^{2}$
}

\begin{abstract}
Résumé. Nous étudions l'effet d'une couche mince rugueuse périodique déposée sur une structure semi-infinie, dans le contexte Helmholtz bi-dimensionnel. Formellement, nous obtenons des conditions de transmission équivalentes à l'ordre 1, par des techniques de type homogénéisation. Suivent alors la résolution du problème du milieu effectif éclairé par une onde plane, et le calcul de la fonction de Green effective; le tout par analyse de Fourier. Dans un deuxième temps, nous considérons le problème de diffraction par un objet pénétrable enfoui dans la structure recouverte par la couche rugueuse. Nous le résolvons par la méthode des éléments finis de frontière, dans le milieu effectif. Des résultats numériques sont présentés. Enfin, le modèle effectif est validé dans le cas d'une couche plate, et l'approximation de Born est utilisée pour tester le code des équations intégrales.
\end{abstract}

Classification Mathématique. 78A25, 78M35, 78A45, 78M15.

Reçu le 30 novembre, 2010. Révisé le 27 février, 2013.

Publication en ligne le 30 Juillet 2013.

\section{INTRODUCTION}

En photonique/optique, on considère des structures en couche grande devant la longueur d'onde que nous pouvons approcher par des espaces semi-infinis. Ces structures ont des fonctionnalités d'anti-reflet; elles sont structurées ou contiennent des nanoparticules permettant d'augmenter leurs propriétés. Elles sont souvent protégées par une couche mince de traitement dur. Le dépôt de cette couche est souvent complexe; cette couche mince présente une surface qui n'est pas lisse. L'influence de cette structuration surfacique involontaire sur la fonctionnalité de la structure doit être examinée. Nous rencontrons un second problème qui est la clusterisation des nanoparticules qui forment alors un aggrégat. Détecter la présence de ces aggrégats est nécessaire. Une détection non invasive est particulièrement intéressante. On comprend le parallèle avec la détection de cellules malignes dans une structure comme la peau. Dans cet article, on s'attache à la prise en compte de l'effet de la couche de protection dans la propagation des ondes, pour un problème modèle. Ce problème entre donc dans le cadre des problèmes de diffraction des ondes par des structures rugueuses, sujet aux multiples applications : optique, acoustique, radar, etc.

\footnotetext{
Mots Clés. Electromagnetics, optics, rough layer, asymptotic analysis, effective transmission conditions, scattering, boundary element method.

1 Institut Elie Cartan de Lorraine, Université de Lorraine, UMR CNRS 7502, 57045 Metz Cedex 1, France. jean-baptiste.bellet@univ-lorraine.fr

2 Thales Optronique, 2, Avenue Gay Lussac CS 90502, 78995 Élancourt Cedex, France. gerard.berginc@fr.thalesgroup.com
} 
$\mathrm{Au}$ sein de différentes communautés, physique, ingénierie, ou mathématiques appliquées, de nombreuses méthodes de résolution analytiques et numériques ont vu le jour $[7,8,13,16,20,22-24]$. Certains auteurs se sont également intéressés à des questions d'existence et d'unicité $[6,10]$. Pour le cas de couches minces périodiques, citons particulièrement les méthodes asymptotiques de type homogénéisation [1,12]. L'un des objectifs de notre article est d'appliquer cette technique, de manière formelle, afin d'obtenir un modèle effectif de couche mince rugueuse périodique recouvrant une structure semi-infinie, supposée anisotrope. L'anisotropie provient éventuellement d'un effet d'homogénéisation à une très petite échelle. On obtient ainsi un problème de type Helmholtz dans deux demi-plans, avec à l'interface, en guise de couche, des conditions de transmission de type Leontovich.

Ce type de modèle, original de par sa provenance, entre dans la catégorie des modèles stratifiés infinis. Comme dans $[4,14,15,18,19]$, l'analyse de Fourier tangentielle à l'interface constitue un bon cadre d'étude. En effet, dans le domaine spectral, les calculs sont explicites, et le principe d'absorption limite permet de poser le problème convenablement, en sélectionnant la solution physiquement réalisable. Cette méthode permet le calcul de la solution du problème de la structure effective éclairée par une onde plane, ainsi que la fonction de Green effective. On constate alors, formellement, que l'effet de la couche est une perturbation des coefficients de réflexion/transmission de Fresnel.

Dans un second temps, nous considérons le problème d'un aggrégat enfoui dans la structure semi-infinie, avec pour source d'éclairage, une onde plane dans le milieu extérieur. Formellement, la couche protectrice est interprétée à l'aide des conditions de transmission effectives calculées au préalable. Il s'agit donc d'un problème de transmission dans un obstacle pénétrable, posé dans le milieu effectif, dont on vient de préciser le calcul la fonction de Green, et l'onde qui se propagerait sans obstacle. À partir d'une représentation intégrale simple couche $[3,5,21]$, la méthode des éléments finis de frontière $[17,18]$ est donc appropriée pour la résolution numérique. Nous en écrivons la formulation.

L'étude présentée dans cet article conduit à un code de calcul. Nous présentons donc ensuite des résultats numériques à titre d'illustration. Dans un premier temps, pour des matériaux directement inspirés de la physique. Dans un deuxième temps, pour des paramètres plus académiques, autorisant des variations de perméabilité.

Enfin, nous présentons quelques tests de validation de ce travail. Concernant le modèle effectif, le cas d'une couche plate est intéressant car tous les calculs sont explicites. Formellement, la solution effective coïncide avec la solution exacte à l'ordre 1. Numériquement, on peut également observer que l'erreur d'approximation est d'ordre 2. D'autre part, le code des moments est testé pour un aggrégat de permittivité, en comparant avec l'approximation de Born.

\section{Modèle EfFectif De COuche mince Rugueuse PÉRIOdique}

\subsection{Modèle rugueux}

Un milieu anisotrope rempli le demi-plan inférieur $\mathscr{D}^{-}=\left\{x_{2}<0\right\}$. On suppose que ce milieu est recouvert d'une couche mince périodique $\mathscr{D}_{\xi}^{\mathrm{cl}}=\left\{\left(x_{1}, x_{2}\right): 0<x_{2}<\xi f\left(x_{1} / \xi\right)\right\}$, où $f>0$ est une fonction 1-périodique, et $\xi>0$ est paramètre désignant la période de la couche et l'ordre de son épaisseur. Le milieu extérieur est $\mathscr{D}_{\xi}^{+}=\left\{x_{2}>\xi f\left(x_{1} / \xi\right)\right\}$. Le tout constitue un diélectrique homogène dans chaque partie du domaine : l'inverse de la perméabilité et la permittivité sont respectivement

$$
A_{\xi}(x)=\frac{1}{\mu^{+}} \mathbb{1}_{\mathscr{D}_{\xi}^{+}}(x)+\frac{1}{\mu^{\mathrm{cl}}} \mathbb{1}_{\mathscr{D}_{\xi}^{\mathrm{cl}}}(x)+A^{-} \mathbb{1}_{\mathscr{D}}(x), \quad \varepsilon_{\xi}(x)=\varepsilon^{+} \mathbb{1}_{\mathscr{D}_{\xi}^{+}}(x)+\varepsilon^{\mathrm{cl}} \mathbb{1}_{\mathscr{D}_{\xi}^{\mathrm{cl}}}(x)+\varepsilon^{-} \mathbb{1}_{\mathscr{D}}-(x),
$$

où $\mathbb{1}$. est la fonction indicatrice, $A^{-}$est une matrice constante symétrique définie positive, et $\mu^{+}, \mu^{\mathrm{cl}}, \varepsilon^{+}, \varepsilon^{\mathrm{cl}}$, $\varepsilon^{-}>0$ sont des constantes réelles. Voir la Figure 1 pour une illustration. 


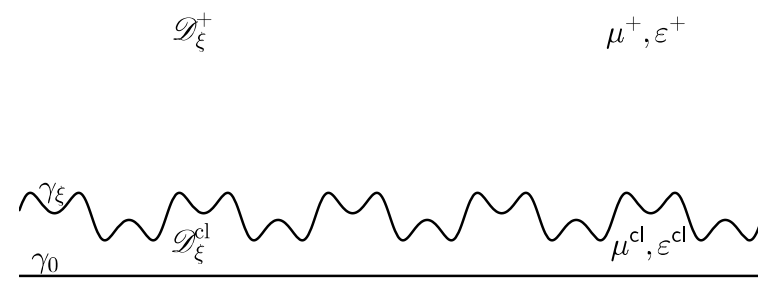

Figure 1. Demi-plan anisotrope séparé de la partie supérieure par une couche mince périodique.

Quitte à effectuer une renormalisation, nous supposerons que $A^{-}$est de la forme $A^{-}=\left(\begin{array}{c}s t \\ t 1\end{array}\right)$. La couche est

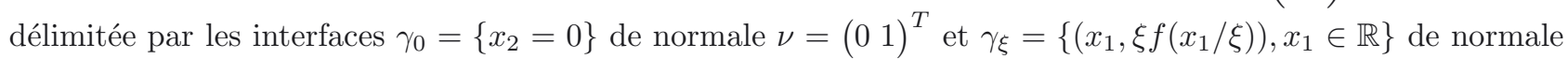
$\nu_{x_{1}}=\frac{1}{\sqrt{1+f^{\prime}\left(x_{1} / \xi\right)^{2}}}\left(-f^{\prime}\left(x_{1} / \xi\right) 1\right)^{T}$ au point d'abscisse $x_{1}$.

Nous étudions ce milieu en régime basse fréquence : la pulsation $\omega>0$ des ondes est supposée vérifier $\omega \xi$ petit devant 1 . De façon équivalente, nous supposerons que $\xi$ est petit devant la longueur d'onde de chacun des milieux.

Dans cette première section, on supposera que le milieu est éclairé par une onde plane dans la partie supérieure $\mathscr{D}_{\xi}^{+}$du domaine $: u_{\text {inc }}(x)=\mathrm{e}^{\mathrm{i} k^{+} \hat{\theta} \cdot x}$, de nombre d'onde $k^{+}=\omega \sqrt{\varepsilon^{+} \mu^{+}}$, et d'angle d'incidence $\hat{\theta}=\left(\hat{\theta}_{1}, \hat{\theta}_{2}\right)$ avec $\hat{\theta}_{2}<0$ et $|\hat{\theta}|=1$. Sa longueur d'onde est $\lambda^{+}:=2 \pi / k^{+}$.

Le problème considéré ici est l'étude de la propagation de l'onde résultante $u_{\xi}$ dans le milieu :

$$
\begin{cases}\frac{1}{\mu^{+}} \Delta u_{\xi}+\omega^{2} \varepsilon^{+} u_{\xi}=0, & \text { dans } \mathscr{D}_{\xi}^{+}, \\ \frac{1}{\mu^{\mathrm{cl}}} \Delta u_{\xi}+\omega^{2} \varepsilon^{\mathrm{cl}} u_{\xi}=0, & \text { dans } \mathscr{D}_{\xi}^{\mathrm{cl}}, \\ \nabla \cdot A^{-} \nabla u_{\xi}+\omega^{2} \varepsilon^{-} u=0, & \text { dans } \mathscr{D}^{-} \\ {\left.\left[u_{\xi}\right]\right|_{\gamma_{0}}=0,\left.\left[\partial_{\nu_{A_{\xi}}} u_{\xi}\right]\right|_{\gamma_{0}}=0, \operatorname{sur} \gamma_{0},} \\ {\left.\left[u_{\xi}\right]\right|_{\gamma_{\xi}}=0,\left.\left[\partial_{\nu_{A_{\xi}}} u_{\xi}\right]\right|_{\gamma_{\xi}}=0, \operatorname{sur} \gamma_{\xi},}\end{cases}
$$

où l'on note : $\left.[u]\right|_{\gamma_{0}}=\left.u\right|_{\gamma_{0}^{+}}-\left.u\right|_{\gamma_{0}^{-}}$, respectivement $\left.\left[\partial_{\nu_{A_{\xi}}} u\right]\right|_{\gamma_{0}}=\left.\frac{1}{\mu^{+}} \partial_{x_{2}} u\right|_{\gamma_{0}^{+}}-\left.(t 1) \nabla u\right|_{\gamma_{0}^{-}}$, le saut de $u$, resp. de sa dérivée conormale, à travers $\gamma_{0}$; et $\left.[u]\right|_{\gamma_{\xi}}=\left.u\right|_{\gamma_{\xi}^{+}}-\left.u\right|_{\gamma_{\xi}^{-}}$, resp. $\left.\left[\partial_{\nu_{A_{\xi}}} u\right]\right|_{\gamma_{\xi}}=\left.\frac{1}{\mu^{+}} \nu \cdot \nabla u\right|_{\gamma_{\xi}^{+}}-\left.\frac{1}{\mu^{\mathrm{cl}}} \nu \cdot \nabla u\right|_{\gamma_{\xi}^{-}}$, le saut de $u$, resp. de sa dérivée conormale, à travers $\gamma_{\xi}$.

Pour que le problème (2.1) soit bien posé mathématiquement, il faut lui ajouter des conditions de type conditions de radiation. Ces conditions, sur le comportement de la solution, sélectionnent la solution acceptable physiquement. Ici, d'une part, l'onde réfléchie $u_{\xi}-u_{\text {inc }}$ dans le milieu supérieur $\mathscr{D}_{\xi}^{+}$doit être montante, et l'onde transmise $u_{\xi}$ dans le milieu inférieur $\mathscr{D}^{-}$doit être descendante. D'autre part, comme dans [19], la fonction de Green risque d'avoir une composante en ondes de surface qui se propagent le long de la couche mince. Il faudrait connaitre le comportement asymptotique de ces ondes pour trouver les conditions de radiation correspondantes. Cette question est difficile à cause de l'anisotropie du milieu inférieur; elle reste ouverte. 
Dans la suite de cette section, nous transformons formellement l'équation (2.1) sous une forme effective, sans se préoccuper des conditions de radiation.

\subsection{Calcul asymptotique formel}

\subsubsection{Plan de travail}

En suivant la démarche de $[1,12]$, nous allons obtenir un développement asymptotique formel de la forme suivante :

$$
u_{\xi}(x)=u_{0}(x)+u_{0}^{\mathrm{cl}, \xi}(x)+\xi\left(u_{1}(x)+u_{1}^{\mathrm{cl}, \xi}(x)\right)+\cdots
$$

Les termes $u_{i}$ sont solution d'un problème de Helmholtz dans le domaine sans la couche $\mathscr{D}_{\xi}^{+}$, avec des conditions de saut à l'interface $\gamma_{0}$ entre le demi-plan inférieur $\mathscr{D}^{-}$et le demi-plan supérieur $\mathscr{D}^{+}=\left\{x_{2}>0\right\}$. La forme de ces problèmes est la suivante :

$$
\mathscr{H}\left[F_{0}, G_{0}\right](u):\left\{\begin{array}{lr}
\frac{1}{\mu^{+}} \Delta u+\omega^{2} \varepsilon^{+} u=0, & \text { dans } \mathscr{D}^{+}, \\
\nabla \cdot A^{-} \nabla u+\omega^{2} \varepsilon^{-} u=0, & \text { dans } \mathscr{D}^{-}, \\
{[u]=F_{0},} & \text { sur } \gamma_{0}, \\
\left.\frac{1}{\mu^{+}} \partial_{x_{2}} u\right|_{\gamma_{0}^{+}}-\left.(t 1) \nabla u\right|_{\gamma_{0}^{-}}=G_{0}, & \text { sur } \gamma_{0} .
\end{array}\right.
$$

Les termes $u_{i}^{\mathrm{cl}, \xi}$ sont des correcteurs de couche limite. Ils sont solution de problèmes de Helmholtz posés dans le domaine avec la couche, avec un second membre et des conditions de saut aux interfaces :

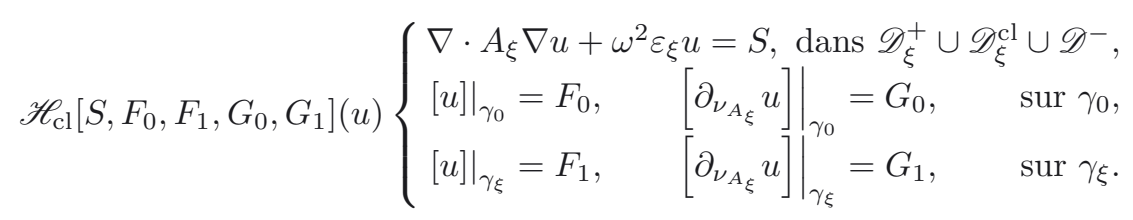

Les correcteurs de couche limite sont définis à partir de problèmes dans la bande de périodicité; ceux-ci leur donnent la particularité de décroître exponentiellement dans la direction normale à l'interface $\gamma_{0}$.

Par remise à l'échelle $y=x / \xi$, la bande de périodicité est $(0,1) \times \mathbb{R}$ et les interfaces $\gamma_{0}$ et $\gamma_{\xi}$ ont pour images respectives $\Gamma_{0}$ et $\Gamma_{1}$. Les problèmes de bande en question ont la forme suivante :

$$
\mathscr{B}\left[F, g_{0}, g_{1}\right](\Psi, \psi):\left\{\begin{array}{l}
\nabla \cdot A \mathscr{Y} \nabla \Psi=F, \quad \text { dans }(0,1) \times \mathbb{R} \backslash \Gamma_{0} \cup \Gamma_{1}, \\
{\left.[\Psi]\right|_{\Gamma_{0}}=0,\left.\quad\left[\partial_{\nu_{A_{Y}}} \Psi\right]\right|_{\Gamma_{0}}=g_{0}, \quad \text { sur } \Gamma_{0},} \\
{\left.[\Psi]\right|_{\Gamma_{1}}=0,\left.\quad\left[\partial_{\nu_{A}} \Psi\right]\right|_{\Gamma_{1}}=g_{1}, \quad \text { sur } \Gamma_{1},} \\
y_{1} \longmapsto \Psi\left(y_{1}, \cdot\right), \quad \text { 1-périodique, } \\
\Psi \longrightarrow 0, y_{2} \rightarrow-\infty ; \quad \Psi \longrightarrow \psi, y_{2} \rightarrow+\infty,
\end{array}\right.
$$

où les inconnues sont la fonction $\Psi$ et sa limite $\psi$. Ici, $A_{\mathscr{Y}}(y)=A(x)$ et les crochets désignent le saut de $\Psi$ ou de sa dérivée conormale $\partial_{\nu_{A \mathscr{Y}}} \Psi(y)=\nabla_{y} \Psi(y)^{T} A \mathscr{Y} \nu_{y}$ à travers les interfaces. Sous des hypothèses techniques sur le second membre $F$, comprenant sa périodicité en $y_{1}$, et la décroissance exponentielle de ses coefficients de Fourier en dehors d'un domaine suffisamment grand $(0,1) \times(-M, M)$, alors, sous la condition de compatibilité des sauts $g_{0}$ et $g_{1}$ :

$$
\int_{(0,1) \times(-M, M)} F \mathrm{~d} y+\int_{\Gamma_{0}} g_{0} \mathrm{~d} \sigma+\int_{\Gamma_{1}} g_{1} \mathrm{~d} \sigma=0,
$$

le problème $\mathscr{B}\left[F, g_{0}, g_{1}\right](\Psi, \psi)$ possède une unique solution $(\Psi, \psi)$; les convergences de $\Psi$ en $+\infty$ et $-\infty$ sont alors exponentielles. Ce résultat est démontré rigoureusement dans [12] lorsque le milieu inférieur est isotrope, preuve que nous avons adaptée dans [9] pour le problème ci-dessus. 


\subsubsection{Développement formel}

Commençons simplement par approcher $u_{\xi}$ par $u_{0}$, solution d'un problème sans la couche : $\mathscr{H}[0,0]\left(u_{0}\right)$.

Celà introduit une erreur d'approximation sur les sauts des dérivées normales à travers les interfaces $\gamma_{0}$ et $\gamma_{\xi}$. Pour les corriger, on introduit le premier correcteur de couche limite $u_{0}^{\mathrm{cl}, \xi}$ tel que :

$$
\mathscr{H}_{\mathrm{cl}}\left[\mathcal{O}(1), \mathcal{O}(\xi), \mathcal{O}(\xi),\left.\left(\frac{1}{\mu^{+}}-\frac{1}{\mu^{\mathrm{cl}}}\right) \nu \cdot \nabla u_{0}\right|_{\gamma_{0}^{+}}+\mathcal{O}(\xi),\left.\left(\frac{1}{\mu^{\mathrm{cl}}}-\frac{1}{\mu^{+}}\right) \nu \cdot \nabla u_{0}\right|_{\gamma_{0}^{+}}+\mathcal{O}(\xi)\right]\left(u_{0}^{\mathrm{cl}, \xi}\right) .
$$

Pour cela, on pose

$$
u_{0}^{\mathrm{cl}, \xi}(x)=\left.\xi\left(\Psi_{0}\left(\frac{x}{\xi}\right)-\psi_{0} \mathbb{1}_{\mathscr{D}}+(x)\right) \cdot \nabla u_{0}\right|_{\gamma_{0}^{+}}
$$

où $\left(\Psi_{0}, \psi_{0}\right)$ est la solution de

$$
\mathscr{B}\left[0,\left(\frac{1}{\mu^{+}}-\frac{1}{\mu^{\mathrm{cl}}}\right) \nu,\left(\frac{1}{\mu^{\mathrm{cl}}}-\frac{1}{\mu^{+}}\right) \nu\right]\left(\Psi_{0}, \psi_{0}\right) .
$$

En approchant $u_{\xi}$ par $u_{0}+u_{0}^{\mathrm{cl}, \xi}$, on commet une erreur sur le saut à travers $\gamma_{0}$. On corrige ce saut à l'aide du terme suivant $\xi u_{1}$, avec $u_{1}$ solution d'un problème dans le domaine sans la couche, de type $\mathscr{H}\left[\left.\psi_{0} \cdot \nabla u_{0}\right|_{\gamma_{0}^{+}}, G_{1}\right]\left(u_{1}\right)$. Le saut $G_{1}$ sera fixé lors de la définition du terme correcteur suivant.

L'estimation de $u_{\xi}$ par $u_{0}+u_{0}^{\mathrm{cl}, \xi}+\xi u_{1}$ donne une erreur sur les sauts des dérivées normales ainsi que dans l'EDP. On introduit alors le second correcteur de de couche limite $u_{1}^{\mathrm{cl}, \xi}$ pour corriger à l'ordre supérieur ces erreurs :

$$
\begin{gathered}
\mathscr{H}_{\mathrm{cl}}\left[\left(-\left(\frac{2}{\mu^{+}} 0\right) \mathbb{1}_{\mathscr{D}_{\xi}^{+}}-\left(\frac{2}{\mu^{\mathrm{cl}}} 0\right) \mathbb{1}_{\mathscr{D}_{\xi}^{\mathrm{cl}}}-(2 r 2 s) \mathbb{1}_{\mathscr{D}^{-}}\right) \nabla_{y} \Psi_{0}(x / \xi) \partial_{x_{1}}\left(\left.\nabla u_{0}\right|_{\gamma_{0}^{+}}\right)\right. \\
-\left.\omega^{2}\left(\varepsilon^{\mathrm{cl}}-\varepsilon^{+} \frac{\mu^{+}}{\mu^{\mathrm{cl}}}\right) u_{0}\right|_{\gamma_{0}^{+}} \mathbb{1}_{\mathscr{D}_{\xi}^{\mathrm{cl}}}+\mathcal{O}(\xi), \\
\begin{array}{r}
\mathcal{O}\left(\xi^{2}\right), \mathcal{O}\left(\xi^{2}\right), \\
\left.\left.\xi s \Psi_{0}\right|_{\Gamma_{0}}(x / \xi) \cdot \partial_{x_{1}}\left(\left.\nabla u_{0}\right|_{\gamma_{0}^{+}}\right)\right|_{\gamma_{0}}+\left.\xi\left(\frac{1}{\mu^{+}}-\frac{1}{\mu^{\mathrm{cl}}}\right) \nu \cdot \nabla u_{1}\right|_{\gamma_{0}^{+}}-\xi G_{1}+\mathcal{O}\left(\xi^{2}\right), \\
\xi\left(\frac{1}{\mu^{\mathrm{cl}}}-\frac{1}{\mu^{+}}\right) f(x / \xi) \nu \cdot\left(\left.\left.\partial_{x_{2} x_{1}}^{2} u_{0}\right|_{\gamma_{0}^{+}}\left(-k^{+^{2}}-\partial_{x_{1}}^{2}\right) u_{0}\right|_{\gamma_{0}^{+}}\right)^{T} \\
+\left.\xi\left(\frac{1}{\mu^{\mathrm{cl}}}-\frac{1}{\mu^{+}}\right) \nu_{1}\left(\left.\Psi_{0}\right|_{\Gamma_{1}}-\psi_{0}\right) \cdot \partial_{x_{1}}\left(\left.\nabla u_{0}\right|_{\gamma_{0}^{+}}\right)\right|_{\gamma_{0}} \\
\left.+\left.\xi\left(\frac{1}{\mu^{\mathrm{cl}}}-\frac{1}{\mu^{+}}\right) \nu \cdot \nabla u_{1}\right|_{\gamma_{0}^{+}}+\mathcal{O}\left(\xi^{2}\right)\right]\left(\xi u_{1}^{\mathrm{cl}, \xi}\right) .
\end{array}
\end{gathered}
$$

Il suffit pour cela de poser

$$
\begin{aligned}
u_{1}^{\mathrm{cl}, \xi}=\xi\left\{\left(\Psi_{0}\left(\frac{x}{\xi}\right)-\psi_{0} \mathbb{1}_{\mathscr{D}^{+}}\right)\right. & \left.\cdot \nabla u_{1}\right|_{\gamma_{0}^{+}}+\left.\left(\Psi_{1}^{(1)}\left(\frac{x}{\xi}\right)-\psi_{1}^{(1)} \mathbb{1}_{\mathscr{D}^{+}}\right) \cdot \partial_{x_{1}}\left(\left.\nabla u_{0}\right|_{\gamma_{0}^{+}}\right)\right|_{\gamma_{0}} \\
+\left(\Psi_{1}^{(2)}\left(\frac{x}{\xi}\right)-\psi_{1}^{(2)} \mathbb{1}_{\mathscr{D}^{+}}\right) & \cdot\left(\begin{array}{c}
\left.\partial_{x_{2} x_{1}}^{2} u_{0}\right|_{\gamma_{0}^{+}} \\
\left(-k^{+2}-\left.\partial_{x_{1}{ }^{2}}^{2} u_{0}\right|_{\gamma_{0}^{+}}\right.
\end{array}\right) \\
& \left.+\left.\omega^{2}\left(\varepsilon^{\mathrm{cl}}-\varepsilon^{+} \frac{\mu^{+}}{\mu^{\mathrm{cl}}}\right)\left(\Psi_{1}^{(3)}\left(\frac{x}{\xi}\right)-\psi_{1}^{(3)} \mathbb{1}_{\mathscr{D}^{+}}\right) u_{0}\right|_{\gamma_{0}^{+}}\right\}
\end{aligned}
$$

où $\left(\Psi_{1}^{(1)}, \psi_{1}^{(1)}\right),\left(\Psi_{1}^{(2)}, \psi_{1}^{(2)}\right)$ et $\left(\Psi_{1}^{(3)}, \psi_{1}^{(3)}\right)$ sont solutions respectives de :

$$
\begin{aligned}
\mathscr{B}\left[\left(-\left(\frac{2}{\mu^{+}} 0\right) \mathbb{1}_{y_{2}>f\left(y_{1}\right)}-\left(\frac{2}{\mu^{\mathrm{cl}}} 0\right) \mathbb{1}_{0<y_{2}<f\left(y_{1}\right)}-(2 r 2 s) \mathbb{1}_{y_{2}<0}\right) \nabla_{y} \Psi_{0},\right. \\
\left.\left.s \Psi_{0}\right|_{\Gamma_{0}}-G_{1}^{(1)},\left(\frac{1}{\mu^{\mathrm{cl}}}-\frac{1}{\mu^{+}}\right) \nu_{1}\left(\left.\Psi_{0}\right|_{\Gamma_{1}}-\psi_{0}\right)\right]\left(\Psi_{1}^{(1)}, \psi_{1}^{(1)}\right),
\end{aligned}
$$




$$
\mathscr{B}\left[0,-G_{1}^{(2)},\left(\frac{1}{\mu^{\mathrm{cl}}}-\frac{1}{\mu^{+}}\right) f \nu\right]\left(\Psi_{1}^{(2)}, \psi_{1}^{(2)}\right), \quad \mathscr{B}\left[-\mathbb{1}_{0<y_{2}<f\left(y_{1}\right)},-G_{1}^{(3)}, 0\right]\left(\Psi_{1}^{(3)}, \psi_{1}^{(3)}\right) .
$$

Pour assurer l'existence et l'unicité de la solution à ces problèmes, $G_{1}^{(1)}, G_{1}^{(2)}$ et $G_{1}^{(3)}$ sont choisis pour satisfaire les conditions de compatibilité de ces équations : $G_{1}^{(1)}=-\left.s \int_{\Gamma_{0}} \Psi_{0}\right|_{\Gamma_{0}} \mathrm{~d} \sigma+\left.\left(\frac{1}{\mu^{+}}-\frac{1}{\mu^{\mathrm{cl}}}\right) \int_{\Gamma_{1}} \nu_{1} \Psi_{0}\right|_{\Gamma_{1}} \mathrm{~d} \sigma, G_{1}^{(2)}=$

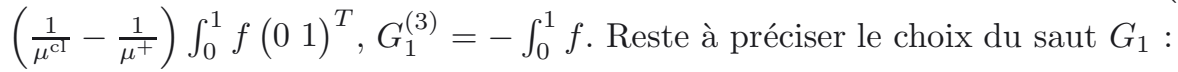

$$
\begin{aligned}
G_{1} & =\left.G_{1}^{(1)} \cdot \partial_{x_{1}}\left(\left.\nabla u_{0}\right|_{\gamma_{0}^{+}}\right)\right|_{\gamma_{0}}+G_{1}^{(2)} \cdot\left(\begin{array}{c}
\left.\partial_{x_{2} x_{1}}^{2} u_{0}\right|_{\gamma_{0}^{+}} \\
\left.\left(-k^{+^{2}}-\partial_{x_{1}}^{2}\right) u_{0}\right|_{\gamma_{0}^{+}}
\end{array}\right)+\left.\omega^{2}\left(\varepsilon^{\mathrm{cl}}-\varepsilon^{+} \frac{\mu^{+}}{\mu^{\mathrm{cl}}}\right) G_{1}^{(3)} u_{0}\right|_{\gamma_{0}^{+}}, \\
& =\left.\left(\varphi_{2} \partial_{x_{1} 2}^{2}+\varphi_{1} \partial_{x_{1} x_{2}}^{2}+\varphi_{0}\right) u_{0}\right|_{\gamma_{0}^{+}},
\end{aligned}
$$

avec

$$
\varphi_{0}=\omega^{2}\left(\varepsilon^{+}-\varepsilon^{\mathrm{cl}}\right) \int_{0}^{1} f, \quad\left(\begin{array}{c}
\varphi_{2}+\left(\frac{1}{\mu^{\mathrm{cl}}}-\frac{1}{\mu^{+}}\right) \int_{0}^{1} f \\
\varphi_{1}
\end{array}\right)=G_{1}^{(1)}
$$

\subsection{Modèle effectif}

Par décroissance exponentielle des correcteurs de couche limite $u_{i}^{\mathrm{cl}, \xi}$ dans le développement (2.2), on peut approcher $u_{\xi}$, excepté à proximité de la couche, par

$$
u_{\xi} \approx u_{0}+\xi u_{1}
$$

Comme cette approximation satisfait : $\mathscr{H}\left[\left.\xi \psi_{0} \cdot \nabla u_{0}\right|_{\gamma_{0}^{+}},\left.\xi\left(\varphi_{2} \partial_{x_{1}{ }^{2}}^{2}+\varphi_{1} \partial_{x_{1} x_{2}}^{2}+\varphi_{0}\right) u_{0}\right|_{\gamma_{0}^{+}}\right]\left(u_{0}+\xi u_{1}\right)$, on approche finalement la solution $u_{\xi}$ de (2.1) par $U$, solution d'un problème sans la couche avec conditions de transmission de type Leontovich à travers l'interface $\gamma_{0}$ :

$$
\left\{\begin{array}{lr}
\frac{1}{\mu^{+}} \Delta U+\omega^{2} \varepsilon^{+} U=0, & \text { dans } \mathscr{D}^{+}, \\
\nabla \cdot A^{-} \nabla U+\omega^{2} \varepsilon^{-} U=0, & \text { dans } \mathscr{D}^{-}, \\
{[U]=\left.\xi \psi_{0} \cdot \nabla U\right|_{\gamma_{0}^{+}},} & \text {sur } \gamma_{0}, \\
\left.\frac{1}{\mu^{+}} \partial_{x_{2}} U\right|_{\gamma_{0}^{+}}-\left.(t 1) \nabla U\right|_{\gamma_{0}^{-}}=\left.\xi\left(\varphi_{2} \partial_{x_{1}{ }^{2}}^{2}+\varphi_{1} \partial_{x_{1} x_{2}}^{2}+\varphi_{0}\right) U\right|_{\gamma_{0}^{+}}, & \text {sur } \gamma_{0}
\end{array}\right.
$$

Plus généralement, dans un problème posé dans le milieu avec la couche, on remplace, formellement, la couche par les conditions de transmission effectives que nous venons d'obtenir. Ceci est le cas pour le problème de la couche éclairée par une onde plane, pour le problème d'un point source dans le milieu inférieur (fonction de Green), mais aussi pour le problème de diffraction par un obstacle enfoui dans le milieu inférieur. Ce sont précisément ces modèles effectifs résultants que nous étudions dans les sections suivantes.

Restent les questions d'existence et d'unicité pour le problème effectif, et surtout la question difficile d'équivalence du modèle effectif et du modèle initial. Pour qu'un problème de type (2.5) soit bien posé, il faut lui ajouter des conditions de radiation. Si cette question est en partie résolue dans la prochaine section à l'aide d'un principe d'absorption limite, il n'est pas évident que les conditions de radiation inconnues du problème initial (2.1) soient identiques. De ce point de vue, il peut y avoir a priori non équivalence des modèles. En particulier, les conditions suffisantes de la section suivante pour assurer l'existence et l'unicité de la solution effective ne sont peut-être pas suffisantes pour bien poser le problème initial. 


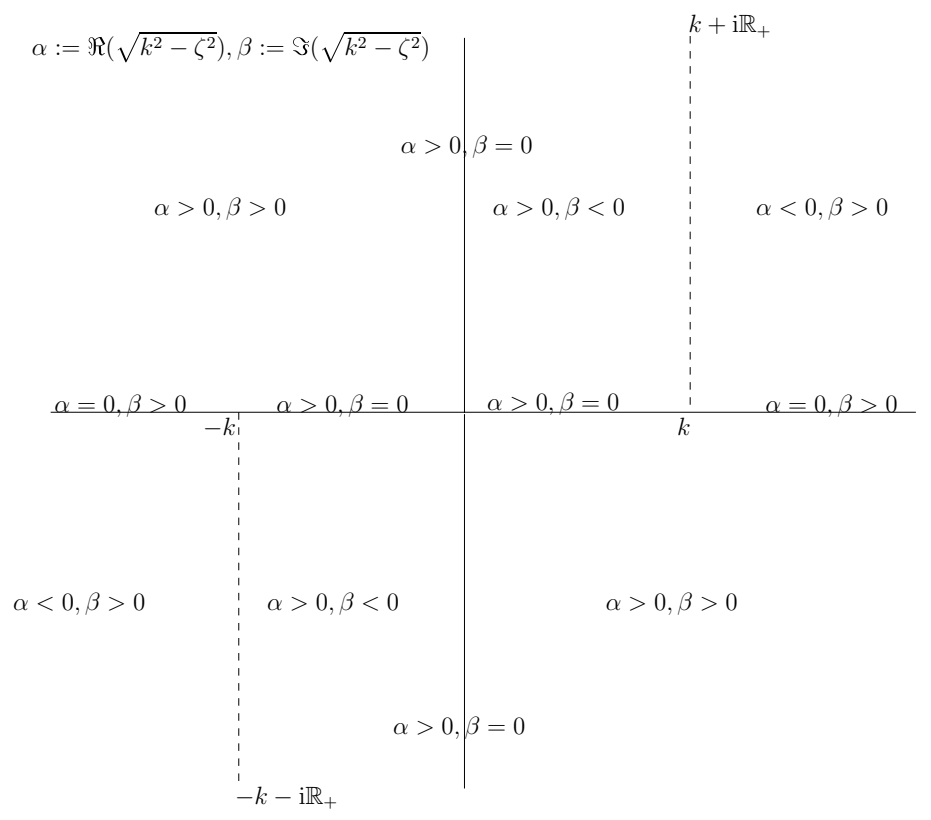

Figure 2. Détermination de $\sqrt{k^{2}-\zeta^{2}}:=\sqrt{k-\zeta} \sqrt{k+\zeta}$ pour $k>0$.

\section{Analyse spectrale Du modèle efFeCtif}

\subsection{Onde incidente plane}

On considère $U$ vérifiant le problème effectif $(2.5)$, avec l'onde incidente plane $u_{\text {inc }}(x)=\mathrm{e}^{\mathrm{i} k^{+} \hat{\theta} \cdot x}$ pour éclairage dans le milieu supérieur. Cette condition d'éclairage nous incite à décomposer l'onde $U$ sous la forme

$$
U(x)= \begin{cases}u_{\text {inc }}(x)+u_{\text {refl }}(x), & x \in \mathscr{D}^{+}, \\ u_{\text {trans }}(x), & x \in \mathscr{D}^{-},\end{cases}
$$

où les ondes $u_{\text {refl }}$ et $u_{\text {trans }}$ seront appelées respectivement onde réfléchie et onde transmise. Par transformation de Fourier en la variable tangentielle $x_{1}$, et de variable spectrale $\zeta$, la version spectrale du problème (2.5) est un système d'équations différentielles ordinaires en $x_{2}$ avec des conditions de raccord :

$$
\begin{cases}\partial_{x_{2}{ }^{2}}^{2} \hat{U}\left(\zeta, x_{2}\right)+\left(k^{+}{ }^{2}-\zeta^{2}\right) \hat{U}\left(\zeta, x_{2}\right)=0, & x_{2}>0, \\ \partial_{x_{2}{ }^{2}}^{2} \hat{U}\left(\zeta, x_{2}\right)+2 \mathrm{i} t \zeta \partial_{x_{2}} \hat{U}\left(\zeta, x_{2}\right)+\left(\omega^{2} \varepsilon^{-}-s \zeta^{2}\right) \hat{U}\left(\zeta, x_{2}\right)=0, & x_{2}<0, \\ \hat{U}\left(\zeta, 0^{+}\right)-\hat{U}\left(\zeta, 0^{-}\right)=\xi \psi_{0} \cdot\left(\mathrm{i} \zeta \hat{U}\left(\zeta, 0^{+}\right) \partial_{x_{2}} \hat{U}\left(\zeta, 0^{+}\right)\right)^{T}, & x_{2}=0, \\ \frac{1}{\mu^{+}} \partial_{x_{2}} \hat{U}\left(\zeta, 0^{+}\right)-(t 1)\left(\mathrm{i} \zeta \hat{U}\left(\zeta, 0^{-}\right) \partial_{x_{2}} \hat{U}\left(\zeta, 0^{-}\right)\right)^{T} & \\ =\xi\left(\left(\varphi_{0}-\zeta^{2} \varphi_{2}\right) \hat{U}\left(\zeta, 0^{+}\right)+\mathrm{i} \zeta \varphi_{1} \partial_{x_{2}} \hat{U}\left(\zeta, 0^{+}\right)\right) & x_{2}=0 .\end{cases}
$$

La résolution de ce système va faire intervenir des racines carrées à définir proprement. Posons $d=\operatorname{det} A^{-}$, $k^{-}=\omega \sqrt{\frac{\varepsilon^{-}}{d}}, \lambda^{+}(\zeta)=\sqrt{{k^{+}}^{2}-\zeta^{2}}$ et $\lambda^{-}(\zeta)=\sqrt{d} \sqrt{k^{-2}-\zeta^{2}}$, où les racines carrées sont déterminées comme suit. On pose, pour $z \neq 0$ d'argument $-\frac{\pi}{2}<\arg z<\frac{3 \pi}{2}, \sqrt{z}=\sqrt{|z|} \mathrm{e}^{\frac{i}{2} \arg z}$, et on pose pour $k>0, \sqrt{k^{2}-\zeta^{2}}=$ $\sqrt{k-\zeta} \sqrt{k+\zeta}$. Cette détermination (Fig. 2) a été choisie telle que $\sqrt{k^{2}-.^{2}}$ est analytique sur $\mathbb{C}$ privé des branches de coupe $k+\mathrm{i} \mathbb{R}^{+}$et $-k-\mathrm{i} \mathbb{R}^{+}$, et telle que pour $\zeta \in \mathbb{R}, \sqrt{k^{2}-\zeta^{2}}$ est dans le premier quadrant. Sous 
ces notations, les équations différentielles signifient que les ondes réfléchie et transmise sont de la forme :

$$
\begin{aligned}
\hat{u}_{\text {refl }}\left(\zeta, x_{2}\right) & =\alpha^{+}(\zeta) \mathrm{e}^{\mathrm{i} \lambda^{+}(\zeta) x_{2}}+\beta^{+}(\zeta) \mathrm{e}^{-\mathrm{i} \lambda^{+}(\zeta) x_{2}}, \quad x_{2}>0, \\
\hat{u}_{\text {trans }}\left(\zeta, x_{2}\right) & =\mathrm{e}^{-\mathrm{i} t \zeta x_{2}}\left(\alpha^{-}(\zeta) \mathrm{e}^{\mathrm{i} \lambda^{-}(\zeta) x_{2}}+\beta^{-}(\zeta) \mathrm{e}^{-\mathrm{i} \lambda^{-}(\zeta) x_{2}}\right), \quad x_{2}<0 .
\end{aligned}
$$

Il reste ainsi à déterminer les quatre inconnues $\alpha^{+}(\zeta), \alpha^{-}(\zeta), \beta^{+}(\zeta), \beta^{-}(\zeta)$, à partir des deux conditions de raccord. Ceci est un problème sous-déterminé et confirme que le problème (2.5) est mal posé si l'on ne précise pas en plus des conditions de radiation (sur le comportement des ondes $u_{\text {refl }}$ et $u_{\text {trans }}$ ).

Comme dans [18], on lève cette indétermination dans le domaine spectral à l'aide d'un principe d'absorption limite. Ainsi, remplaçons formellement dans les expressions précédemment obtenues les nombres d'onde $k^{+}$et $k^{-}$par $k_{\eta}^{+}=k^{+}+$i $\eta$ et $k_{\eta}=k^{-}+$i $\eta$, où $\eta>0$ est un petit paramètre. Dans ce cas, pour $\zeta \in \mathbb{R}$, les racines carrées $\lambda_{\eta}^{+}(\zeta)$ et $\lambda_{\eta}^{-}(\zeta)$ sont à partie imaginaire strictement positive, et donc les composantes en $\mathrm{e}^{-\mathrm{i} \lambda_{\eta}^{+}(\zeta) x_{2}}$ et $\mathrm{e}^{\mathrm{i} \lambda \lambda_{\eta}^{-}(\zeta) x_{2}}$ sont non bornées quand $x_{2}$ tend respectivement vers $+\infty$ et $-\infty$. Physiquement, il est impossible d'avoir de telles composantes non bornées, et donc, on les élimine par la condition $\beta_{\eta}^{+}(\zeta)=0$ et $\alpha_{\eta}^{-}(\zeta)=0$. Le principe d'absorption limite consiste à imposer la même condition à la limite $\eta \rightarrow 0$. Comme $\lambda_{\eta}^{+}(\zeta)$ et $\lambda_{\eta}^{-}(\zeta)$ tendent respectivement vers $\lambda^{+}(\zeta)$ et $\lambda^{-}(\zeta)$, on élimine les composantes en $\mathrm{e}^{-\mathrm{i} \lambda^{+}(\zeta) x_{2}}$ et $\mathrm{e}^{\mathrm{i} \lambda^{-}(\zeta) x_{2}}$ par la condition : $\beta^{+}(\zeta)=0$ et $\alpha^{-}(\zeta)=0$. Notons que ce choix revient à choisir $u_{\text {reff }}$ montante et $u_{\text {trans }}$ descendante.

Pour déterminer les deux dernières inconnues, on traduit sous forme d'un système linéaire $2 \times 2$ les deux conditions de raccord :

$$
\left[\begin{array}{cc}
1-\mathrm{i} \xi c_{1}\left(\lambda^{+}\right) & -1 \\
\lambda^{+}-\mathrm{i} \xi c_{2}\left(\lambda^{+}\right) \mu^{+} & \lambda^{-} \mu^{+}
\end{array}\right]\left[\begin{array}{l}
\alpha^{+} \\
\beta^{-}
\end{array}\right]=-2 \pi \delta\left(\zeta-k^{+} \hat{\theta}_{1}\right)\left[\begin{array}{c}
1-\mathrm{i} \xi c_{1}\left(k^{+} \hat{\theta}_{2}\right) \\
k^{+} \hat{\theta}_{2}-\mathrm{i} \xi c_{2}\left(k^{+} \hat{\theta}_{2}\right) \mu^{+}
\end{array}\right]
$$

où l'on a posé $c_{1}\left(\lambda^{+}\right)=\psi_{0} \cdot\left(\zeta \lambda^{+}\right)^{T}, c_{2}\left(\lambda^{+}\right)=\varphi_{2} \zeta^{2}+\varphi_{1} \zeta \lambda^{+}-\varphi_{0}$. Le déterminant de ce système s'écrit : $D(\zeta)=D_{0}+\xi D_{1}$, avec $D_{0}\left(\lambda^{+}\right)=\lambda^{-} \mu^{+}+\lambda^{+}$et $D_{1}\left(\lambda^{+}\right)=-\mathrm{i} c_{1}\left(\lambda^{+}\right) \lambda^{-} \mu^{+}-\mathrm{i} c_{2}\left(\lambda^{+}\right) \mu^{+}$. Analysons génériquement ce déterminant $D(\zeta)$, de la forme $D\left(\zeta, \lambda^{+}, \lambda^{-}\right)$. En multipliant par les expressions conjuguées des racines carrées, on obtient

$$
P(\zeta)=D\left(\zeta, \lambda^{+}, \lambda^{-}\right) D\left(\zeta,-\lambda^{+}, \lambda^{-}\right) D\left(\zeta, \lambda^{+},-\lambda^{-}\right) D\left(\zeta,-\lambda^{+},-\lambda^{-}\right)
$$

qui est un polynôme dont les racines contiennent les éventuels zéros de $D$. Le polynôme $P$ se décomposant sous la forme $P=: P_{1}+\mathrm{i} P_{2}$ avec $P_{1}$ et $P_{2}$ des polynômes à coefficients réels, une condition nécessaire pour que $D$ s'annule en un réel est que le résultant de $P_{1}$ et $P_{2}$ soit nul. Or on peut interpréter ce résultant comme un polynôme en la taille de la couche $\xi$. Ceci montre, au moins formellement, qu'excepté peut-être pour un nombre fini de valeurs de $\xi$, le déterminant $D(\zeta)$ ne possède pas de zéros réels.

Ainsi, en excluant ces éventuelles valeurs de $\xi$, le système possède une unique solution que l'on obtient par formules de Cramer : $\alpha^{+}=2 \pi R(\zeta) \delta\left(\zeta-k^{+} \hat{\theta}_{1}\right), \beta^{-}=2 \pi T(\zeta) \delta\left(\zeta-k^{+} \hat{\theta}_{1}\right)$, où les coefficients $R(\zeta)=-\frac{D\left(\zeta, k^{+} \hat{\theta}_{2}, \lambda^{-}\right)}{D\left(\zeta, \lambda^{+}, \lambda^{-}\right)}$ et $T(\zeta)=\left(1-\mathrm{i} \xi c_{1}\left(\lambda^{+}\right)\right) R(\zeta)+\left(1-\mathrm{i} \xi c_{1}\left(k^{+} \hat{\theta}_{2}\right)\right)$ sont dits de réflexion/transmission.

Enfin, on remonte à la solution par transformation de Fourier inverse :

$$
\begin{aligned}
u_{\text {refl }}(x) & =R\left(k^{+} \hat{\theta}_{1}\right) \mathrm{e}^{\mathrm{i} k^{+} \hat{\theta}_{R} x}, \quad x_{2}>0 & & \text { avec } \hat{\theta}_{R}=\left(\hat{\theta}_{1}-\hat{\theta}_{2}\right)^{T}, \\
u_{\text {trans }}(x) & =T\left(k^{+} \hat{\theta}_{1}\right) \mathrm{e}^{\mathrm{i} k^{+} \hat{\theta}_{1} x_{1}} \mathrm{e}^{\mathrm{i} \theta_{T} x_{2}}, \quad x_{2}<0, & & \text { avec } \theta_{T}=-t k^{+} \hat{\theta}_{1}-\lambda^{-}\left(k^{+} \hat{\theta}_{1}\right) .
\end{aligned}
$$

La solution $U$ du problème considéré a donc une décomposition de type Fresnel, avec des coefficients de Fresnel généralisés. L'onde réfléchie est plane. En rappelant que l'on choisit $\lambda^{-}\left(k^{+} \hat{\theta}_{1}\right)=\sqrt{d} \sqrt{k^{-2}-\left(k^{+} \hat{\theta}_{1}\right)^{2}}$ dans le premier quadrant, l'onde transmise est plane si $k^{-2}-\left(k^{+} \hat{\theta}_{1}\right)^{2} \geqslant 0$, et elle décroit exponentiellement sinon. 
Développons formellement en puissances de $\xi$ la solution $U$ de $(2.5)$ que nous venons de calculer, ce qui revient à développer les coefficients de réflexion/transmission :

$$
\begin{aligned}
& R\left(k^{+} \hat{\theta}_{1}\right)=R_{0}+\xi R_{1}+\mathcal{O}\left(\xi^{2}\right), \quad T\left(k^{+} \hat{\theta}_{1}\right)=R_{0}-1+\xi\left(R_{1}-\mathrm{i} c_{1}\left(-k^{+} \hat{\theta}_{2}\right)-\mathrm{i} c_{1}\left(k^{+} \hat{\theta}_{2}\right)\right), \\
& \quad \operatorname{avec} R_{0}=-\frac{D_{0}\left(k^{+} \hat{\theta}_{1}, k^{+} \hat{\theta}_{2}, \lambda^{-}\left(k^{+} \hat{\theta}_{1}\right)\right)}{D_{0}\left(k^{+} \hat{\theta}_{1},-k^{+} \hat{\theta}_{2}, \lambda^{-}\left(k^{+} \hat{\theta}_{1}\right)\right)}, R_{1}=-\frac{D_{1}\left(k^{+} \hat{\theta}_{2}\right) D_{0}\left(-k^{+} \hat{\theta}_{2}\right)-D_{0}\left(k^{+} \hat{\theta}_{2}\right) D_{1}\left(-k^{+} \hat{\theta}_{2}\right)}{D_{0}\left(k^{+} \hat{\theta}_{1},-k^{+} \hat{\theta}_{2}, \lambda^{-}\left(k^{+} \hat{\theta}_{1}\right)\right)^{2}} .
\end{aligned}
$$

Identifions, au moins formellement, avec le développement (2.4) de la solution du problème avec couche (2.1). Les termes d'ordre 0 conduisent à la solution $u_{0}$ du problème sans couche. Les termes perturbatifs d'ordre 1 représentent l'effet $u_{1}$ de la couche rugueuse.

\subsection{Fonction de Green}

De manière analogue aux calculs précédents, nous allons calculer la fonction de Green $G$ associée au modèle effectif (2.5), pour un point source $y$ dans le milieu inférieur $\mathscr{D}^{-}$:

$$
\left\{\begin{array}{lr}
\frac{1}{\mu^{+}} \Delta G(\cdot, y)+\omega^{2} \varepsilon^{+} G(\cdot, y)=0, & \text { dans } \mathscr{D}^{+}, \\
\nabla \cdot A^{-} \nabla G(\cdot, y)+\omega^{2} \varepsilon^{-} G(\cdot, y)=\delta_{y}, & \text { dans } \mathscr{D}^{-}, \\
{[G]=\left.\xi \psi_{0} \cdot \nabla G\right|_{\gamma_{0}^{+}},} & \text {sur } \gamma_{0}, \\
\left.\frac{1}{\mu^{+}} \partial_{x_{2}} G\right|_{\gamma_{0}^{+}}-\left.(t 1) \nabla G\right|_{\gamma_{0}^{-}}=\left.\xi\left(\alpha_{2} \partial_{x_{1}{ }^{2}}^{2}+\alpha_{1} \partial_{x_{1} x_{2}}^{2}+\alpha_{0}\right) G\right|_{\gamma_{0}^{+}}, & \text {sur } \gamma_{0} .
\end{array}\right.
$$

La démarche employée est proche de [14]. Nous gardons les mêmes notations que précédemment, excepté pour les coefficients de réflexion/transmission. La traduction spectrale des équations de (3.1) est le système d'équations différentielles :

$$
\begin{cases}\partial_{x_{2} 2}^{2} \hat{G}\left(\zeta, x_{2}\right)+\left(k^{+2}-\zeta^{2}\right) \hat{G}\left(\zeta, x_{2}\right)=0, & x_{2}>0 \\ \partial_{x_{2} 2}^{2} \hat{G}\left(\zeta, x_{2}\right)+2 \mathrm{i} t \zeta \partial_{x_{2}} \hat{G}\left(\zeta, x_{2}\right)+\left(\omega^{2} \varepsilon^{-}-s \zeta^{2}\right) \hat{G}\left(\zeta, x_{2}\right)=\mathrm{e}^{-\mathrm{i} y_{1} \zeta} \delta_{y_{2}}\left(x_{2}\right), & x_{2}<0\end{cases}
$$

dont les solutions sont de la forme :

$$
\hat{G}\left(\zeta, x_{2}, y\right)= \begin{cases}\alpha^{+}(\zeta, y) \mathrm{e}^{\mathrm{i} \lambda^{+}(\zeta) x_{2}}+\beta^{+}(\zeta, y) \mathrm{e}^{-\mathrm{i} \lambda^{+}(\zeta) x_{2}}, & x_{2}>0, \\ \mathrm{e}^{-\mathrm{i} t \zeta x_{2}}\left(\alpha^{->}(\zeta, y) \mathrm{e}^{\mathrm{i} \lambda^{-}(\zeta) x_{2}}+\beta^{->}(\zeta, y) \mathrm{e}^{-\mathrm{i} \lambda^{-}(\zeta) x_{2}}\right), & 0>x_{2}>y_{2}, \\ \mathrm{e}^{-\mathrm{i} t \zeta x_{2}}\left(\alpha^{-<}(\zeta, y) \mathrm{e}^{\mathrm{i} \lambda^{-}(\zeta) x_{2}}+\beta^{-<}(\zeta, y) \mathrm{e}^{-\mathrm{i} \lambda^{-}(\zeta) x_{2}}\right), & x_{2}<y_{2},\end{cases}
$$

avec des conditions de raccord en $x_{2}=y_{2}$ :

$$
\left\{\begin{array}{l}
\hat{G}\left(\zeta, y_{2}+, y\right)-\hat{G}\left(\zeta, y_{2}-, y\right)=0 \\
\partial_{x_{2}} \hat{G}\left(\zeta, y_{2}+, y\right)-\partial_{x_{2}} \hat{G}\left(\zeta, y_{2}-, y\right)=\mathrm{e}^{-\mathrm{i} y_{1} \zeta}
\end{array}\right.
$$

La fonction de Green $G$ est choisie montante dans le demi-espace supérieur $\mathscr{D}^{+}$, et descendante dans le demiespace inférieur $\mathscr{D}^{-}: \beta^{+}(\zeta, y)=0$ et $\alpha^{-<}(\zeta, y)=0$. Traduisons les conditions de transmission de (3.1) sous forme de conditions de raccord supplémentaires :

$$
\left\{\begin{aligned}
\hat{G}\left(\zeta, 0^{+}\right)-\hat{G}\left(\zeta, 0^{-}\right)=\xi \psi_{0} \cdot\left(\mathrm{i} \zeta \hat{G}\left(\zeta, 0^{+}\right) \partial_{x_{2}} \hat{G}\left(\zeta, 0^{+}\right)\right)^{T}, & x_{2}=0 \\
\frac{1}{\mu^{+}} \partial_{x_{2}} \hat{G}\left(\zeta, 0^{+}\right)-(t 1)\left(\mathrm{i} \zeta \hat{G}\left(\zeta, 0^{-}\right) \partial_{x_{2}} \hat{G}\left(\zeta, 0^{-}\right)\right)^{T} & \\
=\xi\left(\left(\varphi_{0}-\zeta^{2} \varphi_{2}\right) \hat{G}\left(\zeta, 0^{+}\right)+\mathrm{i} \zeta \varphi_{1} \partial_{x_{2}} \hat{G}\left(\zeta, 0^{+}\right)\right), & x_{2}=0
\end{aligned}\right.
$$

Les quatres conditions de raccord sont équivalentes au système linéaire $4 \times 4$ suivant :

$$
\left[\begin{array}{cccc}
1-\mathrm{i} \xi c_{1} & -1 & -1 & 0 \\
\lambda^{+}-\mathrm{i} \xi c_{2} \mu^{+} & -\lambda^{-} \mu^{+} & \lambda^{-} \mu^{+} & 0 \\
0 & \mathrm{e}^{\mathrm{i} \lambda^{-} y_{2}} & \mathrm{e}^{-\mathrm{i} \lambda^{-} y_{2}} & -\mathrm{e}^{-\mathrm{i} \lambda^{-} y_{2}} \\
0 & \mathrm{i}\left(-t \zeta+\lambda^{-}\right) \mathrm{e}^{\mathrm{i} \lambda^{-} y_{2}} & -\mathrm{i}\left(t \zeta+\lambda^{-}\right) \mathrm{e}^{-\mathrm{i} \lambda^{-} y_{2}} & \mathrm{i}\left(t \zeta+\lambda^{-}\right) \mathrm{e}^{-\mathrm{i} \lambda^{-} y_{2}}
\end{array}\right]\left[\begin{array}{c}
\alpha^{+} \\
\alpha^{->} \\
\beta^{->} \\
\beta^{-<}
\end{array}\right]=\left[\begin{array}{c}
0 \\
0 \\
0 \\
\mathrm{e}^{-\mathrm{i}\left(y_{1} \zeta-t \zeta y_{2}\right)}
\end{array}\right] .
$$


Par formules de Cramer, on résout ce système, puis on trouve :

$$
\hat{G}\left(\zeta, x_{2}, y\right)=-\frac{\mathrm{i}}{2 \lambda^{-}} \begin{cases}\mathrm{e}^{\mathrm{i} t \zeta y_{2}} T \mathrm{e}^{-\mathrm{i}\left(\lambda^{-} y_{2}+y_{1} \zeta\right)} \mathrm{e}^{\mathrm{i} \lambda^{+} x_{2}}, & x_{2}>0, \\ \mathrm{e}^{-\mathrm{i} t \zeta\left(x_{2}-y_{2}\right)}\left(\mathrm{e}^{\mathrm{i}\left(\lambda^{-}\left|x_{2}-y_{2}\right|-y_{1} \zeta\right)}+R \mathrm{e}^{-\mathrm{i}\left(\lambda^{-} y_{2}+y_{1} \zeta\right)} \mathrm{e}^{-\mathrm{i} \lambda^{-} x_{2}}\right), & x_{2}<0,\end{cases}
$$

où les coefficients dits de transmission/réflexion sont : $T=\frac{2 \mu^{+} \lambda^{-}}{D}$ et $R=\left(1-\mathrm{i} \xi c_{1}\right) T-1$. Cette expression contient des pseudo-singularités provenant de l'annulation du déterminant $-2 \mathrm{i} \lambda^{-}(\zeta) D(\zeta)$ du système : la division par $\lambda^{-}$conduit à deux pseudo-pôles, en $\zeta= \pm k^{-}$. Notons l'absence de pôles, par notre hypothèse de non annulation de $D$ sur $\mathbb{R}$. Celà implique que la fonction de Green $G$ n'a pas de composante de type onde de surface. Il reste à remonter à la fonction de Green $G$ par transformation de Fourier inverse :

$$
G(x, y)= \begin{cases}G_{\text {trans }}(x, y), & x_{2}>0, \\ G_{\text {sour }}(x, y)+G_{\text {refl }}(x, y), & x_{2}<0 .\end{cases}
$$

Le terme $G_{\text {sour }}$ provient du terme source $\hat{G}_{\text {sour }}\left(\zeta, x_{2}, y\right)=-\frac{\mathrm{i}}{2 \lambda^{-}} \mathrm{e}^{-\mathrm{i} t \zeta\left(x_{2}-y_{2}\right)} \mathrm{e}^{\mathrm{i}\left(\lambda^{-}\left|x_{2}-y_{2}\right|-y_{1} \zeta\right)}$. Par la représentation de Sommerfeld [2] :

$$
-\frac{\mathrm{i}}{4 \pi} \int_{\mathbb{R}} \frac{\sqrt{d}}{\lambda^{-}(\zeta)} \mathrm{e}^{\mathrm{i}\left(\tilde{x}_{1}-\tilde{y}_{1}\right) \zeta} \mathrm{e}^{\mathrm{i} \frac{\lambda^{-}(\zeta)}{\sqrt{d}}\left|\tilde{x}_{2}-\tilde{y}_{2}\right|} \mathrm{d} \zeta=-\frac{\mathrm{i}}{4} H_{0}^{(1)}\left(k^{-}|\tilde{x}-\tilde{y}|\right), \quad \tilde{x} \neq \tilde{y}
$$

on note que $G_{\text {sour }}$ est la fonction de Green sortante d'un espace libre, dont les paramètres sont ceux du milieu inférieur $\left(A^{-}\right.$et $\left.\varepsilon^{-}\right)$:

$$
G_{\text {sour }}(x, y)=-\frac{\mathrm{i}}{4 \sqrt{d}} H_{0}^{(1)}\left(k^{-}\left|A^{*} x-A^{*} y\right|\right), \quad \text { avec } A^{*}=\left(\begin{array}{ll}
1 & -t \\
0 & \sqrt{d}
\end{array}\right) .
$$

Le terme de réflexion $\hat{G}_{\text {refl }}\left(\zeta, x_{2}, y\right)=-\frac{\mathrm{i}}{2 \lambda^{-}} \mathrm{e}^{-\mathrm{i} t \zeta\left(x_{2}-y_{2}\right)} R \mathrm{e}^{-\mathrm{i}\left(\lambda^{-} y_{2}+y_{1} \zeta\right)} \mathrm{e}^{-\mathrm{i} \lambda^{-} x_{2}}$ se décompose en $\hat{G}_{\text {refl }}=$ $\hat{G}_{\text {reg }}+\hat{G}_{\text {imag. }}$. La partie $\hat{G}_{\text {imag }}=\frac{\mathrm{i}}{2 \lambda^{-}} \mathrm{e}^{-\mathrm{i} t \zeta\left(x_{2}-y_{2}\right)} \mathrm{e}^{-\mathrm{i}\left(\lambda^{-} y_{2}+y_{1} \zeta\right)} \mathrm{e}^{-\mathrm{i} \lambda^{-} x_{2}}$ conduit dans le domaine spatial, par la représentation de Sommerfeld, à une fonction de Green de type image :

$$
G_{\mathrm{imag}}(x, y)=\frac{\mathrm{i}}{4 \sqrt{d}} H_{0}^{(1)}\left(k^{-}\left|\bar{A}^{*} x-A^{*} y\right|\right), \quad \text { avec } \bar{A}^{*}=\left(\begin{array}{cc}
1 & -t \\
0 & -\sqrt{d}
\end{array}\right) .
$$

Enfin, la partie $\hat{G}_{\text {reg }}=-\frac{\mathrm{i}\left(1-\mathrm{i} \xi c_{1}\right) T}{2 \lambda^{-}} \mathrm{e}^{-\mathrm{i} t \zeta\left(x_{2}-y_{2}\right)} \mathrm{e}^{-\mathrm{i}\left(\lambda^{-} y_{2}+y_{1} \zeta\right)} \mathrm{e}^{-\mathrm{i} \lambda^{-} x_{2}}$, et le terme de transmission $\hat{G}_{\text {trans }}\left(\zeta, x_{2}, y\right)=$ $-\frac{\mathrm{i}}{2 \lambda^{-}} \mathrm{e}^{\mathrm{i} t \zeta y_{2}} T \mathrm{e}^{-\mathrm{i}\left(\lambda^{-} y_{2}+y_{1} \zeta\right)} \mathrm{e}^{\mathrm{i} \lambda^{+} x_{2}}$ ne possèdent pas de singularité. Leurs versions spatiales peuvent donc être obtenues numériquement par inversion rapide de la transformée de Fourier (IFFT). À noter la décroissance exponentielle des transformées de Fourier $\hat{G}_{\text {reg }}$ et $\hat{G}_{\text {trans }}$ pour les fréquences $|\zeta|>k^{-}$; ceci permet de les tronquer. Précisons enfin que l'expression obtenue pour la fonction de Green $G$ permet d'obtenir le calcul de son gradient $\nabla G$, quitte là encore à effectuer des IFFT.

Comme pour $U$, si l'on écrit un développement en $\xi$ des coefficients de réflexion/transmission, on peut identifier, formellement, avec les termes d'un développement de type (2.4) pour la fonction de Green du problème avec couche (2.1). Celà permet d'isoler formellement l'effet de la couche.

\section{Modèle effectif D'AGgrégat enfoui}

On considère un aggrégat (ou objet) enfoui dans la partie inférieure du milieu : $D \subset \mathscr{D}^{-}$, de paramètres électromagnétiques $\varepsilon_{D}, \mu_{D}>0$, et de normale unitaire extérieure $\nu_{x}$ (en $x$ ). L'ajout de $D$ dans le milieu périodique (2.1), se traduit formellement à l'aide du modèle effectif (2.5). Il s'agit de résoudre :

$$
\left\{\begin{array}{lr}
\frac{1}{\mu^{+}} \Delta u+\omega^{2} \varepsilon^{+} u=0, & \text { dans } \mathscr{D}^{+}, \\
\nabla \cdot\left(A^{-} \mathbb{1}_{\mathscr{D}-\backslash \bar{D}}+\frac{1}{\mu_{D}} \mathbb{1}_{D}\right) \nabla u+\omega^{2}\left(\varepsilon^{-} \mathbb{1}_{\mathscr{D}-\backslash \bar{D}}+\varepsilon_{D} \mathbb{1}_{D}\right) u=0, & \text { dans } \mathscr{D}^{-}, \\
{[u]=\left.\xi \psi_{0} \cdot \nabla u\right|_{\gamma_{0}^{+}}} & \text {sur } \gamma_{0}, \\
\left.\frac{1}{\mu^{+}} \partial_{x_{2}} u\right|_{\gamma_{0}^{+}}-\left.(t 1) \nabla u\right|_{\gamma_{0}^{-}}=\left.\xi\left(\varphi_{2} \partial_{x_{1}{ }^{2}}^{2}+\varphi_{1} \partial_{x_{1} x_{2}}^{2}+\varphi_{0}\right) u\right|_{\gamma_{0}^{+}}, & \text {sur } \gamma_{0},
\end{array}\right.
$$

la source d'énergie considérée étant l'onde plane $u_{\text {inc }}(x)=\mathrm{e}^{\mathrm{i} k^{+} \hat{\theta} \cdot x}$, dans le milieu supérieur $\mathscr{D}^{+}$. 
Introduisons quelques notations. La solution du problème sans objet (2.5), et la fonction de Green associée (3.1) sont encore notées respectivement $U$ et $G$. La fonction de Green associée à l'objet est $G_{D}(x, y):=-\frac{i}{4} H_{0}^{(1)}\left(\omega \sqrt{\varepsilon_{D} \mu_{D}}|x-y|\right)$. Les potentiels simple couche, associés au milieu de fond, et à l'objet, sont $\mathcal{S} \varphi(x):=\int_{\partial D} G(x, y) \varphi(y) \mathrm{d} \sigma(y), \mathcal{S}_{D} \varphi(x):=\int_{\partial D} G_{D}(x, y) \varphi(y) \mathrm{d} \sigma(y)$. Enfin, posons : $\mathcal{K}^{*} \varphi(x):=$ $\int_{\partial D} \nu_{x} \cdot A^{-} \nabla_{x} G(x, y) \varphi(y) \mathrm{d} \sigma(y), \quad \mathcal{K}_{D}^{*} \varphi(x):=\int_{\partial D} \nu_{x} \cdot \nabla_{x} G_{D}(x, y) \varphi(y) \mathrm{d} \sigma(y)$.

Sous ces notations, la solution $u$ de (4.1) possède la représentation intégrale [3,5] :

$$
u= \begin{cases}U+\mathcal{S} \varphi & \text { dans } \mathscr{D}^{+} \cup\left(\mathscr{D}^{-} \backslash \bar{D}\right), \\ \mathcal{S}_{D} \psi & \text { dans } D\end{cases}
$$

où le couple $(\varphi, \psi) \in L^{2}(\partial D) \times L^{2}(\partial D)$ est l'unique solution du système d'équations intégrales :

$$
\left[\begin{array}{cc}
\mathcal{S} & -\mathcal{S}_{D} \\
\frac{\mathrm{I}}{2}+\mathcal{K}^{*}-\frac{1}{\mu_{D}}\left(-\frac{\mathrm{I}}{2}+\mathcal{K}_{D}^{*}\right)
\end{array}\right]\left[\begin{array}{l}
\varphi \\
\psi
\end{array}\right]=\left[\begin{array}{c}
-U \\
-\nu_{x} \cdot A^{-} \nabla U
\end{array}\right], \text { sur } \partial D
$$

Pour calculer numériquement $u$, par la méthode des éléments finis de frontière, on résout la formulation variationnelle de ce système, posée pour $(\varphi, \psi) \in H^{-1 / 2}(\partial D) \times H^{-1 / 2}(\partial D)$, avec les fonctions tests de la première équation dans $H^{-1 / 2}(\partial D)$ et celles de la deuxième équation dans $H^{1 / 2}(\partial D)$.

Pour la discrétisation, le bord $\partial D$ est approché par un polygône direct $\left(x_{0}, \ldots, x_{N}\right)$, et convenons que les indices sont à comprendre modulo $N+1$ (par exemple $\left.x_{N+1}=x_{0}\right) \cdot H^{-1 / 2}(\partial D)$ est approché de façon conforme par l'espace des fonctions constantes sur chaque morceau $\left(x_{i}, x_{i+1}\right)$, dont une base est l'ensemble des $\kappa_{i}=$ $\frac{1}{\left|x_{i+1}-x_{i}\right|} \mathbb{1}_{\left(x_{i}, x_{i+1}\right)}, 0 \leqslant i \leqslant N$; enfin, $H^{1 / 2}(\partial D)$ est approché de façon conforme par l'espace des fonctions affines sur chaque morceau $\left(x_{i}, x_{i+1}\right)$, dont une base est l'ensemble des $\chi_{i}=\frac{\left|x-x_{i+1}\right|}{\left|x_{i}-x_{i+1}\right|} \mathbb{1}_{\left(x_{i}, x_{i+1}\right)}+\frac{\left|x-x_{i-1}\right|}{\left|x_{i}-x_{i-1}\right|} \mathbb{1}_{\left(x_{i-1}, x_{i}\right)}$, $0 \leqslant i \leqslant N$. En décomposant les approximations de $\varphi$ et $\psi$ dans la base des $\kappa_{i}: \varphi \approx \sum_{i} \Phi_{i} \kappa_{i}, \psi \approx \sum_{i} \Psi_{i} \kappa_{i}$, le système linéaire à résoudre numériquement est donc de la forme :

$$
\left[\begin{array}{cc}
\mathcal{M}[G] & -\mathcal{M}\left[G_{D}\right] \\
\mathcal{N}^{+}\left[A^{-} \nabla_{x} G\right] & -\frac{1}{\mu_{D}} \mathcal{N}^{-}\left[\nabla_{x} G_{D}\right]
\end{array}\right]\left[\begin{array}{l}
\Phi \\
\Psi
\end{array}\right]=\left[\begin{array}{l}
-\mathcal{U} \\
-\mathcal{U}^{\prime}
\end{array}\right]
$$

avec pour $0 \leqslant i, j \leqslant N$ :

$$
\begin{aligned}
\mathcal{M}[g]_{i j} & =\int_{\left(x_{i}, x_{i+1}\right)} \kappa_{i}(x) \int_{\left(x_{j}, x_{j+1}\right)} \kappa_{j}(y) g(x, y) \mathrm{d} \sigma(y) \mathrm{d} \sigma(x), \\
\mathcal{N}^{ \pm}\left[g^{\prime}\right]_{i j} & = \pm \frac{1}{4}\left(\delta_{i, j}+\delta_{i-1, j}\right)+\int_{\left(x_{i-1}, x_{i}, x_{i+1}\right)} \chi_{i}(x) \int_{\left(x_{j}, x_{j+1}\right)} \kappa_{j}(y) \nu_{x} \cdot g^{\prime}(x, y) \mathrm{d} \sigma(y) \mathrm{d} \sigma(x), \\
\mathcal{U}_{i} & =\int_{\left(x_{i}, x_{i+1}\right)} \kappa_{i}(x) U(x) \mathrm{d} \sigma(x), \quad \mathcal{U}_{i}^{\prime}=\int_{\left(x_{i-1}, x_{i}, x_{i+1}\right)} \chi_{i}(x) \nu_{x} \cdot A^{-} \nabla U(x) \mathrm{d} \sigma(x),
\end{aligned}
$$

où, $\delta_{\text {., }}$ est le symbole de Kronecker, et sur chaque $\left(x_{i}, x_{i+1}\right), \nu_{x}=\left(\begin{array}{cc}0 & 1 \\ -1 & 0\end{array}\right) \frac{x_{i+1}-x_{i}}{\left|x_{i+1}-x_{i}\right|}$.

Les éléments de la matrice du système sont donc des intégrales, dont il reste à commenter le calcul. On évalue les intégrales de fonctions régulières par une méthode de quadrature de Gauss-Legendre à deux points. Aussi, faut-il prêter attention aux singularités des intégrandes; les termes concernés sont : les trois diagonales $j=i-1, i, i+1$ de $\mathcal{M}[G]$ et $\mathcal{M}\left[G_{D}\right]$, ainsi que les quatre diagonales $j=i-2, i-1, i, i+1$ de $\mathcal{N}^{+}\left[A^{-} \nabla_{x} G\right]$ et $\mathcal{N}^{-}\left[\nabla_{x} G_{D}\right]$. Pour ces termes, il faut intégrer la partie singulière de façon exacte après l'avoir isolée. Pour plus de détails sur ce type de calculs, nous renvoyons aux annexes de [18]. Enfin, précisons que $\mathcal{M}\left[G_{D}\right]$ est symétrique, ce qui réduit le nombre de termes à calculer. (En revanche, $\mathcal{M}[G], \mathcal{N}^{+}\left[A^{-} \nabla_{x} G\right]$ et $\mathcal{N}^{-}\left[\nabla_{x} G_{D}\right]$ sont a priori non symétriques). 

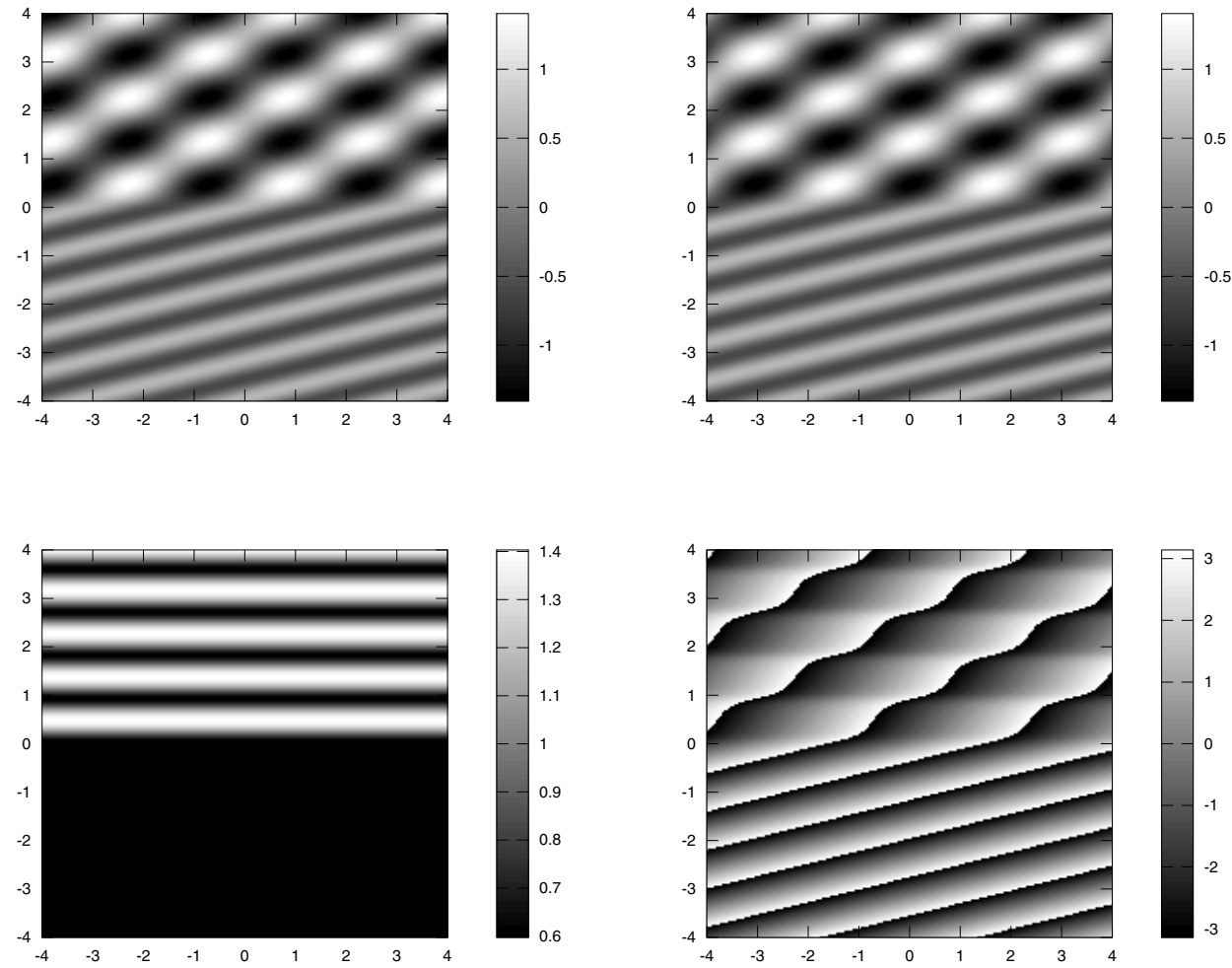

Figure 3. Solution $U$ du problème effectif pour l'exemple physique. Sont représentés, de gauche à droite : parties réelle et imaginaire (en haut), module et argument (en bas).

\section{RÉSultats numéRiques}

\subsection{Exemple physique}

Le milieu supérieur est l'air, de paramètres électromagétiques (relatifs) $\varepsilon^{+}=\mu^{+}=1$. La sonde employée pour l'expérience, de type laser, est supposée émettre une onde plane de longueur d'onde $\lambda^{+}=1.54 \mu \mathrm{m}$, et d'angle d'incidence $\theta=-\pi / 3=-1.04720$. La pulsation considérée est donc $\omega=2 \pi / 1.54=4.07999(/ \mu \mathrm{m})$. Le milieu inférieur semi-infini est supposé périodique, contenant des nanoparticules. La cellule élémentaire est un carré de quartz de $20 \mathrm{~nm}$ de côté, contenant un disque de rutile de rayon $7.5 \mathrm{~nm}$. Le quartz Si02 et le rutile Ti02 sont non magnétiques, et ont pour indices de réfraction respectifs 1.52783 et 2.48317. Ainsi, la perméabilité est $\mu^{-}=1$, donc $A^{-}=\mathrm{I}$, et par effet d'homogénéisation, nous supposerons que la permittivité est la moyenne des permittivités dans la cellule de périodicité $: \varepsilon^{-}=2.48317^{2} p+1.52783^{2}(1-p)=4.02713$, où $p=\frac{\pi 7.5^{2}}{20^{2}}=0.441786$ est la proportion de rutile. La couche mince qui protège le milieu inférieur est un diélectrique de paramètres $\varepsilon^{\mathrm{cl}}=2$ et $\mu^{\mathrm{cl}}=1$. Sa grandeur caractéristique est $\xi=\lambda^{+} / 2^{4}=0.0962500 \mu \mathrm{m}$. Ici, concernant la fonction périodique $f$ qui décrit la forme de la couche, comme les matériaux sont non magnétiques, seule comptera sa moyenne, que nous choisissons égale à $\int_{0}^{1} f=1$.

Pour l'obtention du modèle effectif de la couche, comme $\mu^{+}=\mu^{\mathrm{cl}}$, alors on trouve facilement $\left(\Psi_{0}, \psi_{0}\right)=(0,0)$, puis $\varphi_{1}=\varphi_{2}=0$, et $\varphi_{0}=\omega^{2}\left(\varepsilon^{+}-\varepsilon^{\mathrm{cl}}\right) \int f=-16.6463$. Puis, on peut calculer la solution du modèle effectif $(2.5)$ : voir Figure 3. Sur la Figure 4, nous avons représenté la fonction de Green effective $G(\cdot, y)$ pour le point source $y=(0,-3)$. Pour le calcul des IFFT, nous avons ici choisi pour fenêtre de fréquence $|\zeta| \leqslant 1.1 k^{-}$, discrétisée avec $2^{11}$ points équidistants. 

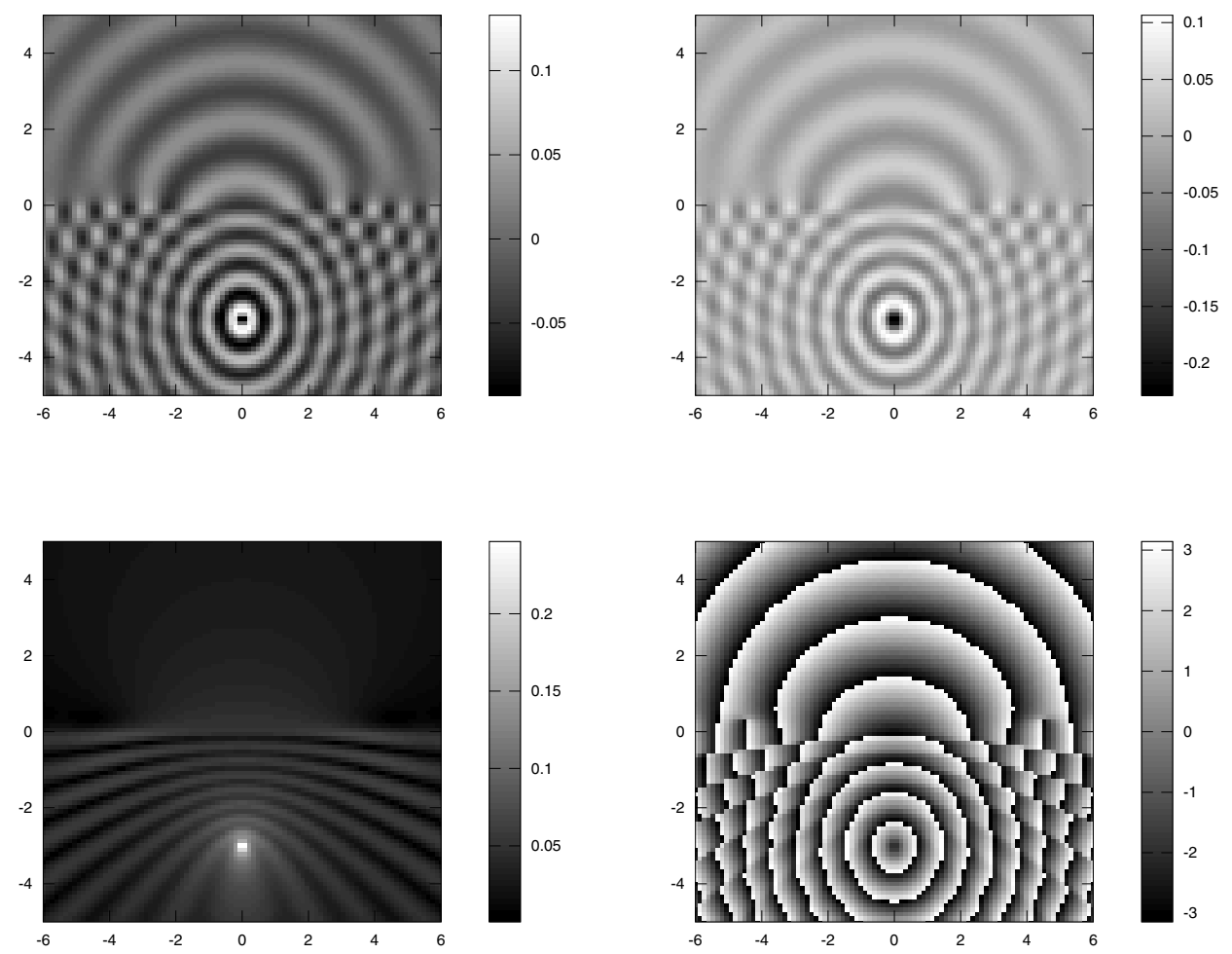

Figure 4. Fonction de Green effective $G$ pour l'exemple physique. Sont représentés, de gauche à droite : parties réelle et imaginaire (en haut), module et argument (en bas).

Enfin, l'inclusion $D$ enfouie est un aggrégat de rutile dont les paramètres sont $\varepsilon_{D}=2.48317^{2}=6.16613$ et $\mu_{D}=1$. Nous choisissons une étoile, paramétrée par :

$$
x(t)=\left(\begin{array}{c}
0 \\
-4
\end{array}\right)+(1+0.15 \cos 5 t)\left(\begin{array}{c}
\cos t \\
\sin t
\end{array}\right) .
$$

On la discrétise en maillant uniformément l'intervalle de paramétrisation $[0,2 \pi]$, avec $N+2=202$ points. Sur la Figure 5, on représente l'onde diffractée par l'étoile $\mathcal{S} \varphi$ dans le milieu effectif; puis, sur la Figure 6 , on représente l'onde totale $u$, solution du problème effectif (4.1) avec aggrégat. Ici, les IFFT ont été calculées avec $2^{10}$ points, sur la fenêtre $|\zeta| \leqslant 1.1 k^{-}$.

\subsection{Exemple académique}

L'étude de cet article va au-delà du modèle physique précédent. Nous montrons un exemple plus général, avec des variations de perméablitié, et un milieu inférieur anisotrope. Les paramètres que l'on ne mentionne pas explicitement sont repris identiques à l'exemple précédent.

La perméabilité de la couche est $\mu^{\mathrm{cl}}=0.8$. Dans le milieu inférieur, le tenseur est $A^{-}=\left(\begin{array}{cc}1.3 & 0.2 \\ 0.2 & 1\end{array}\right)$. Cette fois, la forme de la couche joue un rôle dans le modèle effectif : choisissons $f\left(y_{1}\right)=\frac{1}{4} \sin \left(3 \cos \left(2 \pi y_{1}+1\right)\right)+1$, représentée sur la Figure 7. Le calcul des conditions de transmission effectives ne peut plus se faire entièrement à la main. Nous les avons obtenues en résolvant par éléments finis les problèmes de bande portant sur les deux composantes de $\left(\Psi_{0}, \psi_{0}\right)$ : voir Figure 8. On obtient : $\varphi_{0}=-16.6463, \varphi_{1}=-2.11505 \cdot 10^{-6}, \varphi_{2}=-0.246031$, 

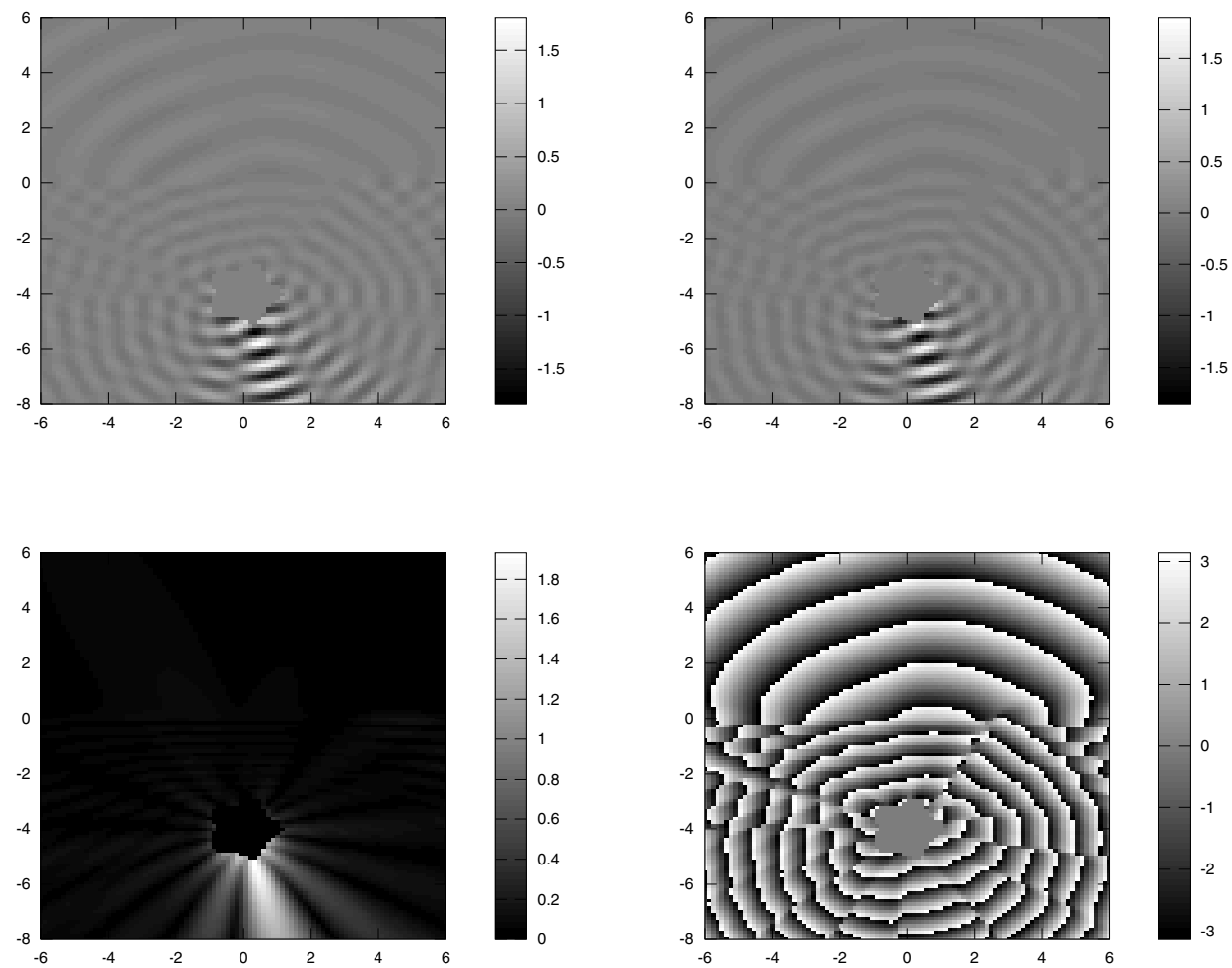

Figure 5. Onde diffractée par l'aggrégat $\mathcal{S} \varphi$ dans le milieu effectif pour l'exemple physique. Sont représentés, de gauche à droite : parties réelle et imaginaire (en haut), module et argument (en bas).

et $\psi_{0}=\left(1.46007 \times 10^{-5},-0.203175\right)$. Sur les Figures 9 et 10 , on représente respectivement la fonction de Green effective (3.1), ainsi que la solution du problème effectif (4.1) avec aggrégat.

\section{Quelques Tests De VAlidation}

\subsection{Cas d'une couche plate : exact versus effectif}

Pour une couche plate, i.e. $f$ constante, la solution $u_{\xi}$ du problème (2.1) de la structure semi-infinie recouvert d'une couche, éclairée par une onde plane, se calcule explicitement. En effet, par des calculs analogues aux calculs spectraux de la Section 3, ou en s'inspirant de [11], on obtient $u_{\xi}$ sous la forme :

$$
u_{\xi}= \begin{cases}u_{\text {inc }}+\tilde{R}_{12} \mathrm{e}^{\mathrm{i} \lambda^{+}\left(x_{2}-2 \xi f\right)} \mathrm{e}^{\mathrm{i} k^{+} \hat{\theta}_{1} x_{1}}, & x_{2}>\xi f, \\ A_{2}\left(\mathrm{e}^{-\mathrm{i} \lambda^{c 1} x_{2}}+R_{23} \mathrm{e}^{\mathrm{i} \lambda^{\mathrm{cl}} x_{2}}\right) \mathrm{e}^{\mathrm{i} k^{+} \hat{\theta}_{1} x_{1}}, & \xi f>x_{2}>0, \\ A_{3} \mathrm{e}^{-\mathrm{i} t k^{+} \hat{\theta}_{1} x_{2}} \mathrm{e}^{-\mathrm{i} \lambda^{-} x_{2}} \mathrm{e}^{\mathrm{i} k^{+} \hat{\theta}_{1} x_{1}}, & 0>x_{2},\end{cases}
$$

avec, ici, $\lambda^{+}=\lambda^{+}\left(k^{+} \hat{\theta}_{1}\right), \lambda^{-}=\lambda^{-}\left(k^{+} \hat{\theta}_{1}\right), \lambda^{\mathrm{cl}}=\sqrt{\omega^{2} \varepsilon^{\mathrm{cl}} \mu^{\mathrm{cl}}-\left(k^{+} \hat{\theta}_{1}\right)^{2}}$ (dans le premier quadrant), et pour coefficients (de réflexion/transmission et d'amplitude) $: R_{12}=\frac{\mu^{\mathrm{cl}} \lambda^{+}-\mu^{+} \lambda^{\mathrm{cl}}}{\mu^{\mathrm{cl}} \lambda^{+}+\mu^{+} \lambda^{\mathrm{cl}}}, R_{21}=-R_{12}, R_{23}=\frac{\lambda^{\mathrm{cl}}-\lambda^{-} \mu^{\mathrm{cl}}}{\lambda^{\mathrm{cl}}+\lambda^{-} \mu^{\mathrm{cl}}}$, $T_{12}=R_{12}+1, T_{21}=R_{21}+1, T_{23}=R_{23}+1, \tilde{R}_{12}=R_{12}+\frac{T_{12} R_{23} T_{21} \mathrm{e}^{\mathrm{i} 2 \lambda} \lambda^{\mathrm{cl}} \xi f}{1-R_{21} R_{23} \mathrm{e}^{\mathrm{i} 2 \lambda{ }^{\mathrm{cl}} \xi f}}, A_{2}=\frac{T_{12} \mathrm{e}^{\mathrm{i}\left(-\lambda^{+}+\lambda^{\mathrm{cl}}\right) \xi f}}{1-R_{21} R_{23} \mathrm{e}^{\mathrm{i} 2 \lambda^{\mathrm{c}} \xi f}{ }_{\xi f}}$ et $A_{3}=T_{23} A_{2}$. 

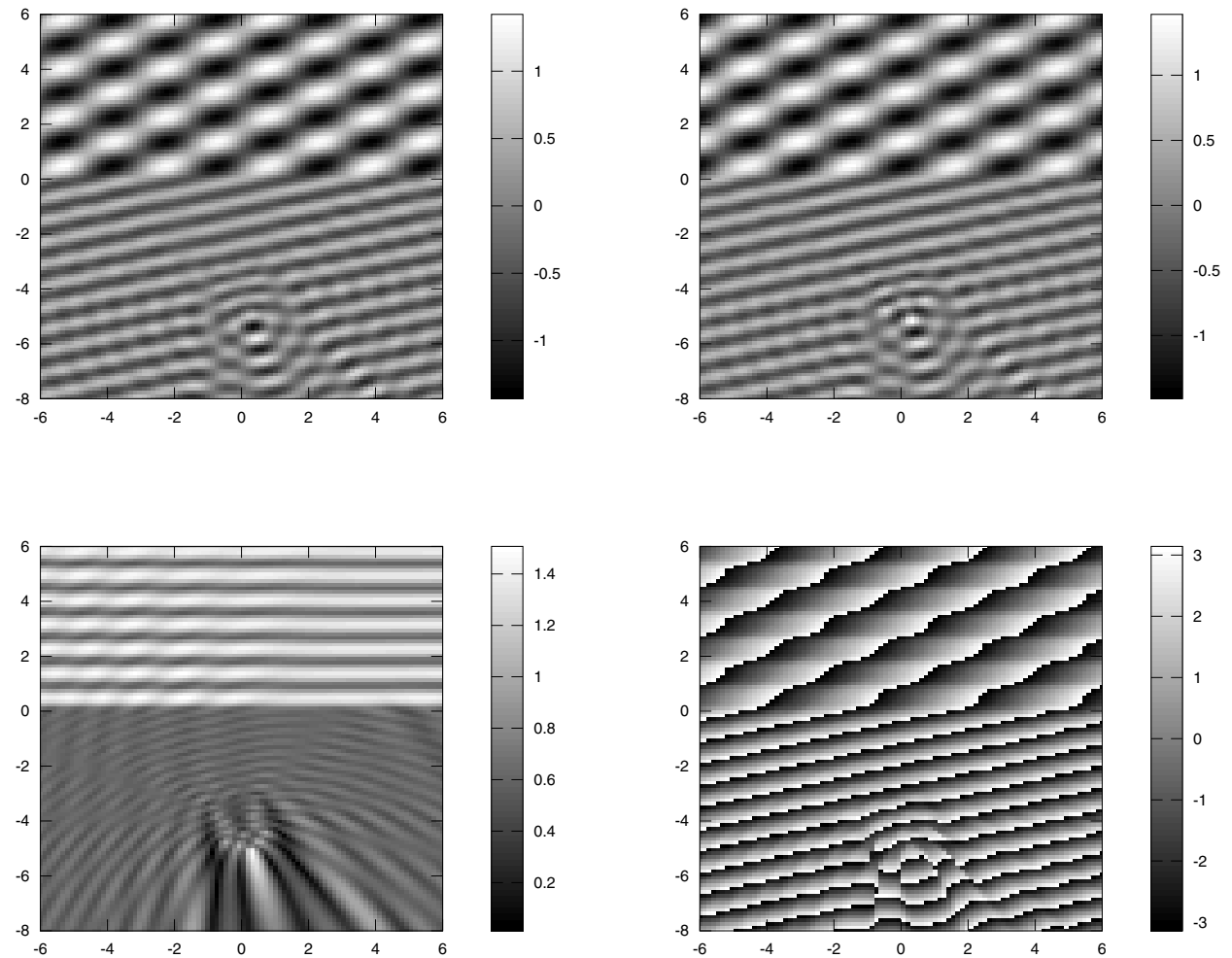

Figure 6. Solution $u$ du problème effectif (4.1) avec aggrégat pour l'exemple physique. Sont représentés, de gauche à droite : parties réelle et imaginaire (en haut), module et argument (en bas).

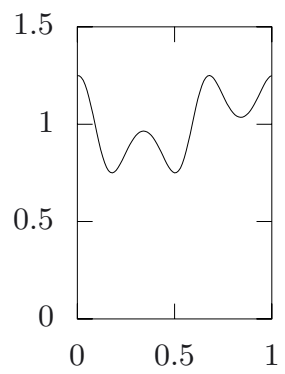

Figure 7. Représentation de la forme $f$ de la couche, sur une période.

D'autre part, le calcul de $U$, solution du problème effectif (2.5) est lui aussi entièrement explicite. On peut en effet vérifier que la solution $\left(\Psi_{0}, \psi_{0}\right)$ du problème de bande $(2.3)$ a pour première composante $\Psi_{0}^{1}=0=\psi_{0}^{1}$ et pour deuxième composante $\Psi_{0}^{2}=\left(\frac{\mu^{\mathrm{cl}}}{\mu^{+}}-1\right)\left(f \mathbb{1}_{x_{2}>f}+x_{2} \mathbb{1}_{f>x_{2}>0}\right), \psi_{0}^{2}=\left(\frac{\mu^{\mathrm{cl}}}{\mu^{+}}-1\right) f$. On en déduit $\varphi_{0}=\omega^{2}\left(\varepsilon^{+}-\varepsilon^{\mathrm{cl}}\right) f$, $\varphi_{1}=0$, et $\varphi_{2}=-\left(\frac{1}{\mu^{\mathrm{cl}}}-\frac{1}{\mu^{+}}\right) f$, puis $U$ par la Section 3 .

À l'aide d'un logiciel de calcul formel, nous avons développé en puissances de $\xi$ le terme de réflexion de la solution exacte $u_{\xi}$ et celui de son approximation effective $U$. Les termes d'ordre 0 et 1 en $\xi$ coïncident. De même pour le terme de transmission. Formellement, la solution effective coïncide donc avec la solution exacte, à l'ordre 1 . 

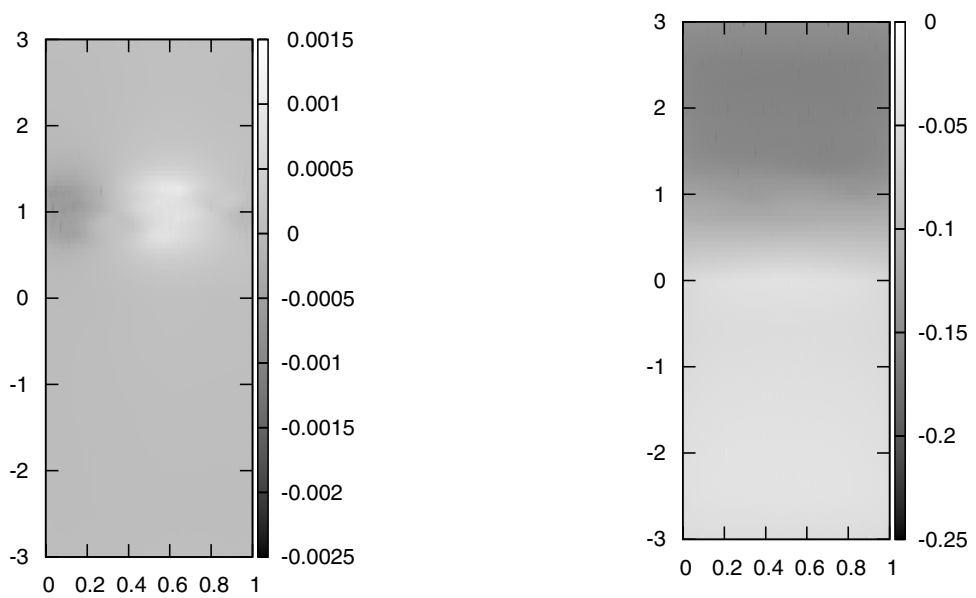

Figure 8. Représentation des deux composantes de $\Psi_{0}$, solution d'un problème dans la bande de périodicité; résultats obtenus par éléments finis avec Freefem++, dans $(0,1) \times(-3,3)$.
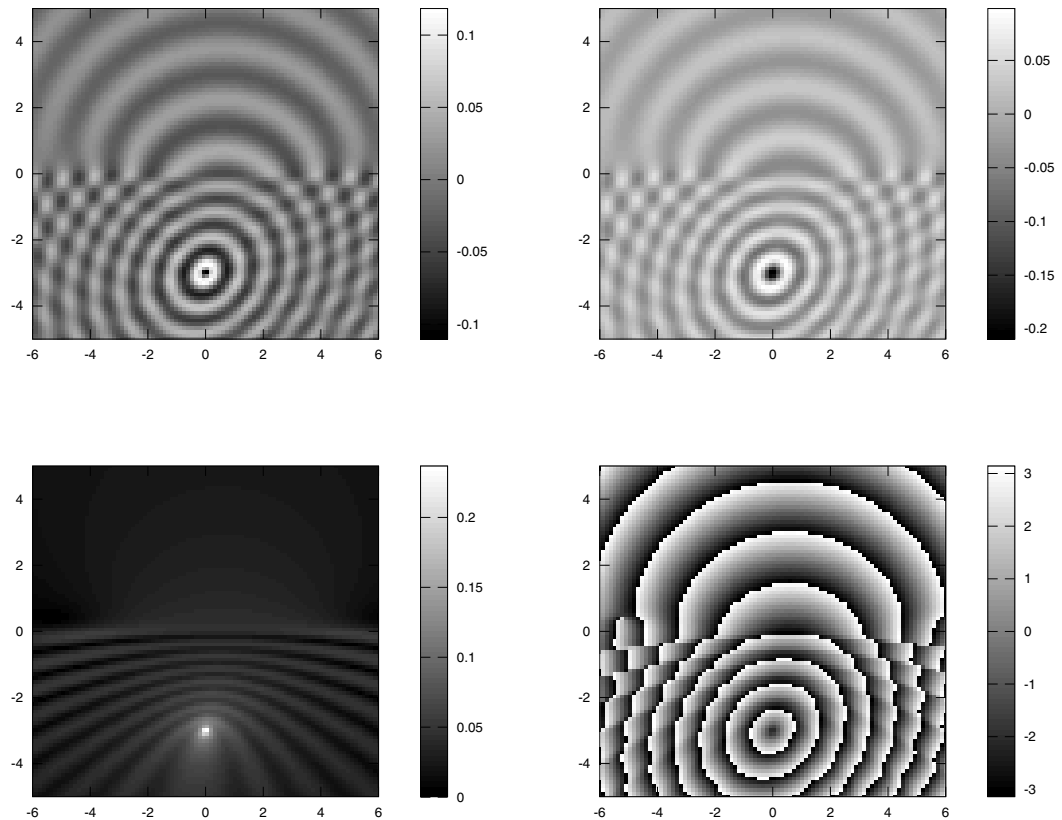

Figure 9. Fonction de Green effective $G$ pour l'exemple académique. Sont représentés, de gauche à droite : parties réelle et imaginaire (en haut), module et argument (en bas). 

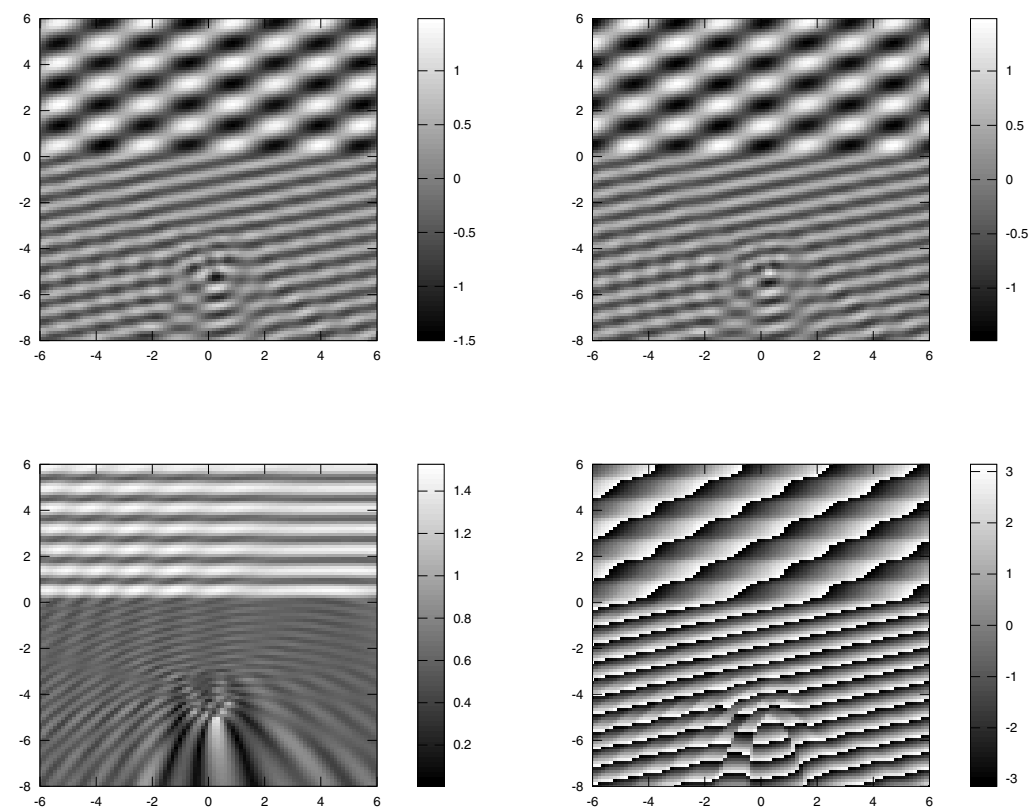

FiguRE 10. Solution $u$ du problème effectif avec aggrégat pour l'exemple académique. Sont représentés, de gauche à droite : parties réelle et imaginaire (en haut), module et argument (en bas).

Enfin, constatons numériquement l'ordre d'approximation du modèle. Pour cela, calculons la solution effective et la solution exacte, pour différents ordres d'épaisseur de couche $: \xi=\frac{\lambda^{+}}{2^{2}}, 0 \leqslant i \leqslant 14$. Ici, les paramètres choisis pour la simulation sont les suivants : $\mu^{+}=2, \mu^{\mathrm{cl}}=5, A^{-}=\left(\begin{array}{ll}2 & 1 \\ 1 & 1\end{array}\right), \varepsilon^{+}=1, \varepsilon^{\mathrm{cl}}=1.2, \varepsilon^{-}=0.6, f\left(y_{1}\right)=0.8$, $\omega=3$ et $\theta=-\pi / 3$. Pour chaque valeur de $\xi$, on calcule l'onde réfléchie $u_{\text {refl }}$, respectivement l'onde transmise $u_{\text {trans }}$, sur un segment de longueur $4 \lambda^{+}$, portion de la droite $x_{2}=5 \lambda^{+}$, respectivement $x_{2}=-5 \lambda^{+}$. $\grave{A}$ titre d'exemple, on a tracé ces quantités sur la Figure 11, pour $\xi=\lambda^{+} / 2^{4}$. Puis, nous calculons la norme 2 de l'erreur, pour l'onde réfléchie, et l'onde transmise. Sur la Figure 12, on trace le logarithme binaire de ces erreurs, en fonction de $\xi=\frac{\lambda^{+}}{2^{i}}$ (ou plutôt de $i$ ). Comme on obtient des courbes à l'allure de droites de pente -2 , l'ordre numérique de l'erreur du modèle effectif est 2 .

\subsection{Aggrégat de permittivité : méthode des moments versus approximation de Born}

Soit un aggrégat enfoui dans la structure semi-infinie. On suppose qu'il s'agit d'une inclusion de permittivité, dont la permittivité est une petite variation de la permittivité de la structure. Ainsi, si les paramètres électromagnétiques de la structure sont $A^{-}$et $\varepsilon^{-}=1$, ceux de l'aggrégat sont $A^{-}$, et $\varepsilon_{D}=1+q$, avec $q$ petit devant 1. On éclaire le milieu effectif (soit, la structure recouverte de la couche) à l'aide d'une onde plane. D'une part, l'onde diffractée par l'aggrégat $\mathcal{S} \varphi(x)$ est calculée par la méthode des moments. D'autre part, sous ces hypothèses, l'approximation de Born [3] consiste à approcher l'onde diffractée par :

$$
u_{\text {Born }}(x)=-\omega^{2} \int_{D} q(y) G(x, y) U(y) \mathrm{d} y,
$$

où $G$ et $U$ sont respectivement la fonction de Green effective, et la solution effective dans le milieu sans aggrégat, calculées dans la Section 3. Cette expression est très pratique à implémenter : il suffit de calculer une intégrale 

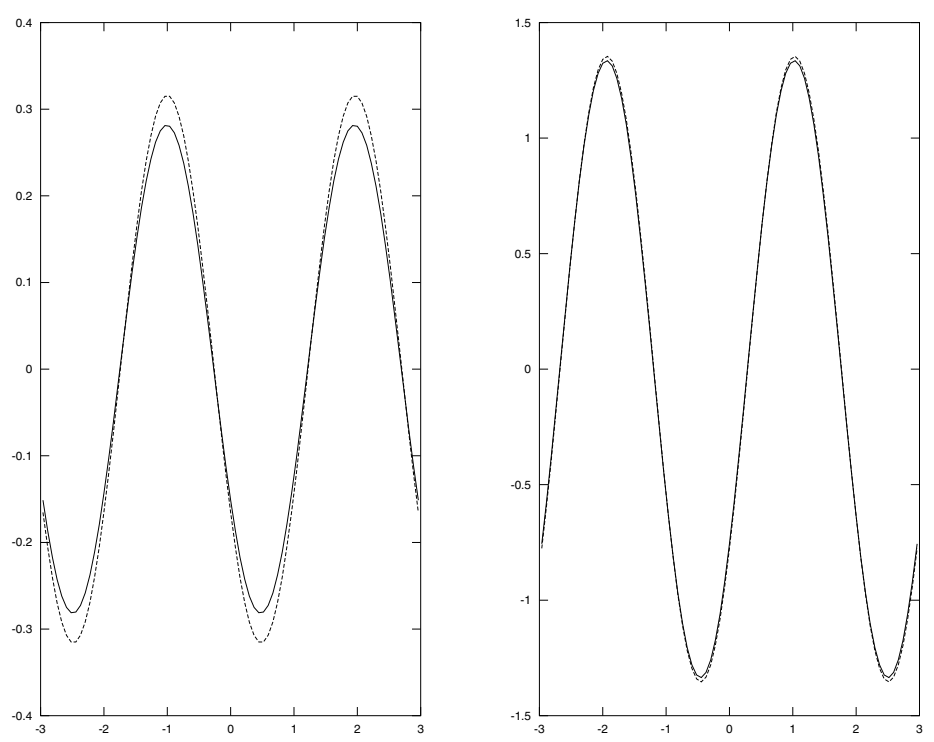

FiguRE 11. Ondes réfléchie $u_{\text {refl }}$ (à gauche) et onde transmise $u_{\text {trans }}$ (à droite) dans le cas d'une couche plate. Le calcul est effectué de façon exacte (traits pleins), et selon le modèle effectif (traits pointillés). Les ondes sont évaluées sur une portion de la droite $x_{2}=5 \lambda^{+}$, respectivement $x_{2}=-5 \lambda^{+}$.
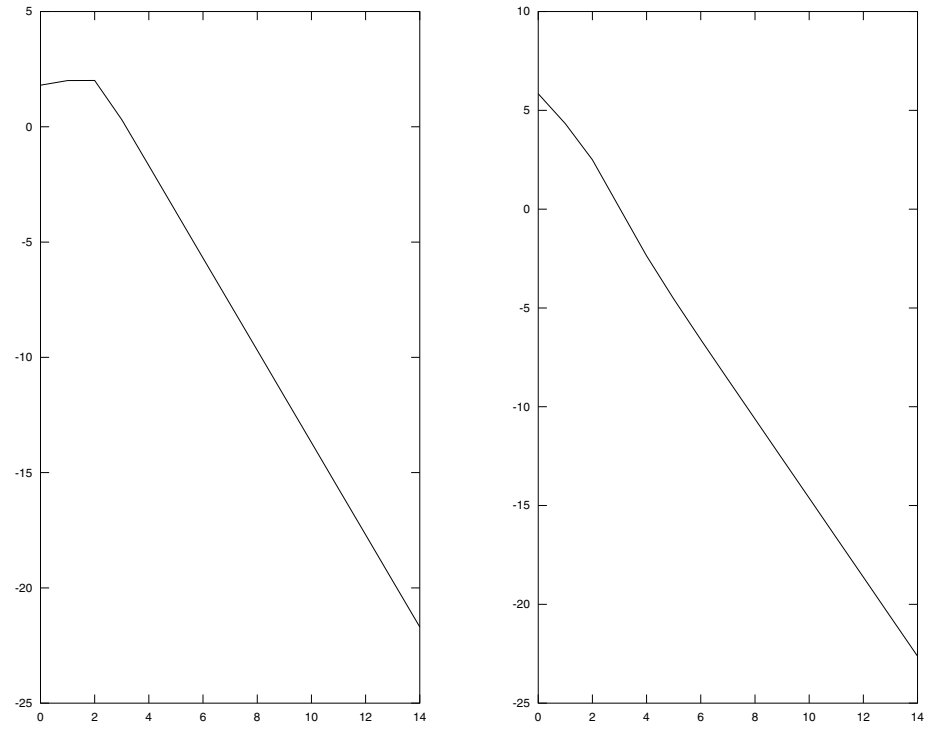

FIGURE 12. Erreurs quadratiques du modèle effectif pour une couche plate, pour l'onde réfléchie (à gauche) et pour l'onde transmise (à droite). Le logarithme des erreurs est tracé en fonction de l'ordre d'épaisseur de la couche. 

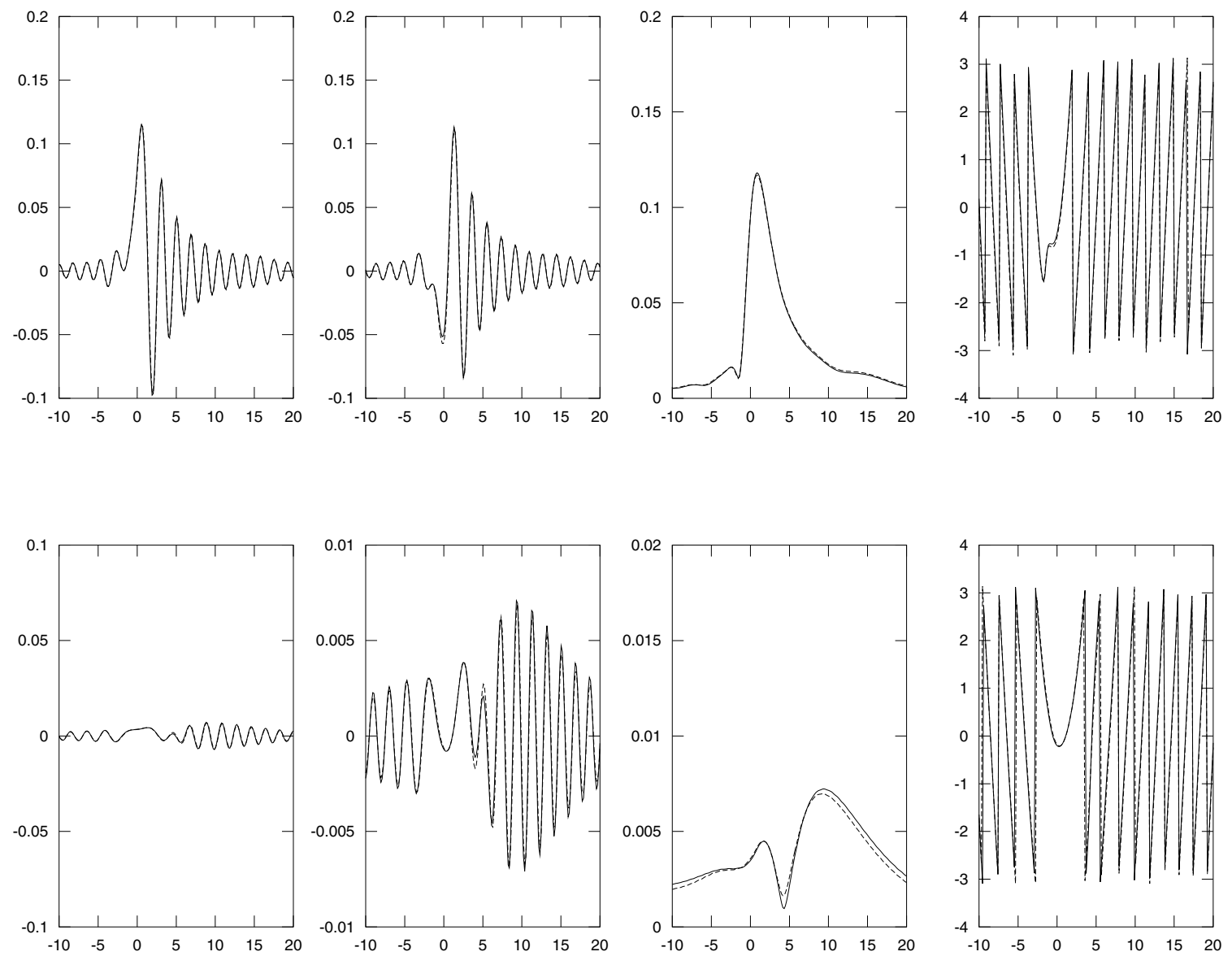

Figure 13. Comparaison de l'onde diffractée par l'aggrégat, calculée par la méthode des moments (traits pleins) et par l'approximation de Born (traits pointillés). Le calcul est effectué dans la structure pour la première ligne, dans le milieu extérieur pour la deuxième. Sont représentés, de la gauche vers la droite : parties réelles et imaginaires, modules, arguments.

d'une fonction régulière. Pour notre test, nous intégrons sur un rectangle contenant l'objet, à partir d'une formule de quadrature composite. Sur chaque sous-rectangle, on utilise une formule déduite (par produit tensoriel) de la formule de Gauss-Legendre à deux points.

On calcule l'onde diffractée par les deux méthodes, et l'on compare les résultats, sur la Figure 13. Ici, les paramètres du modèle sont : $\mu^{+}=1, \mu^{\mathrm{cl}}=0.8, A^{-}=\left(\begin{array}{cc}1.3 & 0.2 \\ 0.2 & 1\end{array}\right), \varepsilon^{+}=1, \varepsilon^{\mathrm{cl}}=2, \varepsilon^{-}=1, f\left(y_{1}\right)=$ $\frac{1}{4} \sin \left(3 \cos \left(2 \pi y_{1}+1\right)\right)+1, \omega=4.07999, \theta=-\pi / 3$, et $\xi=\lambda^{+} / 2^{4}$. L'aggrégat est de frontière (5.1), et de paramètres $A^{-}$et $\varepsilon_{D}=1+q$, avec $q=0.04$. Pour la méthode des moments, l'objet est discrétisé en 202 points, et les IFFT sont calculées avec $2^{10}$ points, sur la fenêtre $|\zeta| \leqslant 1.1 k^{-}$. Pour l'approximation de Born, l'intégrale est calculée sur le carré $(-1.2,1.2) \times(-5.2,-2.8)$ (en étendant $q$ par 0 hors de l'objet); pour la formule composite, les côtés des sous-carreaux sont de l'ordre du dixième de la longueur d'onde. Le calcul est effectué sur une portion des droites $x_{2}=-6$ et $x_{2}=1$, ce qui permet d'avoir la comparaison dans la structure semi-infinie, et dans le milieu extérieur. La comparaison est concluante quant à la validité du code des moments : les courbes sont quasi-confondues. 
Remerciements. Les auteurs remercient chaleureusement Habib Ammari pour ses conseils et suggestions.

\section{RÉFÉRENCES}

[1] T. Abboud and H. Ammari, Diffraction at a curved grating: Tm and te cases, homogenization. J. Math. Anal. Appl. 202 (1996) 995-1026.

[2] M. Abramowitz and I.A. Stegun, Handbook of Mathematical Functions. Dover Publications (1965).

[3] H. Ammari, An Introduction to Mathematics of Emerging Biomedical Imaging. Springer (2008).

[4] H. Ammari, E. Iakovleva and D. Lesselier, A music algorithm for locating small inclusions buried in a half-space from the scattering amplitude at a fixed frequency. Multiscale Model. Simul. 3 (2005) 597-628.

[5] H. Ammari and H. Kang. Reconstruction of small inhomogeneities from boundary measurements. Springer Verlag (2004).

[6] T. Arens and T. Hohage, On radiation conditions for rough surface scattering problems. IMA J. Appl. Math. 70 (2005) 839-847.

[7] G. Bao, Z. Chen and H. Wu, Adaptive finite-element method for diffraction gratings. JOSA A 22 (2005) 1106-1114.

[8] G. Bao, D. Dobson and J. Cox, Mathematical studies in rigorous grating theory. JOSA A 12 (1995) $1029-1042$.

[9] J.-B. Bellet, Identification par imagerie laser d'un objet dissimulé - Aspects mathématiques et numériques. Ph.D. thesis, École Polytechnique, Palaiseau (2011).

[10] S. Chandler-Wilde, C. Ross and B. Zhang, Scattering by infinite one-dimensional rough surfaces. Proc. Royal Soc. London. Ser. A : Math. Phys. Engrg. Sci. 455 (1999) 3767-3787.

[11] W. Chew, Waves and fields in inhomogenous media. IEEE Press (1999).

[12] I. Ciuperca, M. Jai and C. Poignard, Approximate transmission conditions through a rough thin layer. The case of the periodic roughness. Eur. J. Appl. Math. (2009).

[13] J. DeSanto, G. Erdmann, W. Hereman and M. Misra, Theoretical and computational aspects of scattering from periodic surfaces : one-dimensional transmission interface. Waves Random Media 11 (2001) 425-453.

[14] M. Durán, R. Hein and J.-C. Nédélec, Computing numerically the greens function of the half-plane helmholtz operator with impedance boundary conditions. Numer. Math. 107 (2007) 295-314.

[15] M. Durán, I. Muga and J. Nédélec, The helmholtz equation in a locally perturbed half-space with non-absorbing boundary. Arch. Ration. Mech. Anal. 191 (2009) 143-172.

[16] T. Gaylord and M. Moharam, Analysis and applications of optical diffraction by gratings. IEEE 73 (1985) 894-937.

[17] W. C. Gibson, The Method of Moments in Electromagnetics. Chapman and Hall/CRC (2008).

[18] R. Hein, Green's functions and integral equations for the Laplace and Helmholtz operators in impedance half-spaces. Ph.D. thesis, École Polytechnique, Palaiseau (2010).

[19] C. Jerez-Hanckes and J.-C. Nédélec, Asymptotics for Helmholtz and Maxwell solutions in 3-D open waveguides. Technical report, ETH, Zürich (2010).

[20] L. Li, J. Chandezon, G. Granet and J. Plumey, Rigorous and efficient grating-analysis method made easy for optical engineers. Appl. Optics 38 (1999) 304-313.

[21] J.-C. Nédélec, Acoustic and Electromagnetic Equations. Springer (2001).

[22] E. Popov and M. Nevière, Grating theory: new equations in fourier space leading to fast converging results for tm polarization. JOSA A 17 (2000) 1773-1784.

[23] A. Soubret, Diffusion des ondes électromagnétiques par des milieux et des surfaces aléatoires : étude des effets cohérents dans le champ diffusé. Ph.D. thesis, Université de la Méditerranée - Aix-Marseille II (2001).

[24] Y. Wu and Y. Lu, Analyzing diffraction gratings by a boundary integral equation neumann-to-dirichlet map method. JOSA A 26 (2009) 2444-2451. 